\title{
A Fieldmap-Driven Few-Channel Shim Coil Design for MRI of the Human Brain
}

\author{
Bruno Pinho Meneses \& Alexis Amadon \\ Universite Paris-Saclay, CEA, CNRS, BAOBAB, NeuroSpin, 91191 \\ Gif-sur-Yvette, France. \\ E-mail: bruno.pinho-meneses@cea.fr
}

August 2020

\begin{abstract}
We exploit the inter-subject similarity of inhomogeneous static magnetic field patterns arising in the human brain under MRI examination to design a small set of shim coils providing performance equivalent to numerous coils based on high-order Spherical Harmonics corrections. A hundred brain $B_{0}$-maps were first collected at $3 \mathrm{~T}$. Ideal subject-specific electric current density stream functions are then computed with low power constraints, on a cylindrical surface. This step is repeated over tens of brain maps so that a Principal Component Analysis can be applied to the stream functions; the main components result in the small set of coils. Both 50-subject hold-out and 10-fold cross-validation are employed to evaluate consistency of the proposed system performance over a posteriori subjects. Simulations show that only 3 cylindrical coils manage to capture the principal magnetic field profiles in the human brain, thus providing a better static field inhomogeneity mitigation than that obtained from 16 unlimited-power high-order Spherical Harmonics coils, with inhomogeneity greatly reduced in the pre-frontal cortex compared to $2^{\text {nd }}$-order shimmed baseline field acquisitions. The approach provides a very reduced channel count system for mitigating complex $B_{0}$-inhomogeneity patterns. Thus, a compact, cost-effective system could be conceived and driven by relatively low-budget electronics. The method should therefore have a strong impact in both Ultra-High and portable low-field MRI/MRS. Moreover, this technique can be applied to the design of shim coils addressing anatomies other than the brain.
\end{abstract}

Keywords: $B_{0}$ inhomogeneity, human brain shimming, MRI, shim coil design, stream functions, Ultra-High-Field MRI, whole brain shimming.

\section{Introduction}

As commercial and research MRI equipment move towards Ultra-High Field (UHF) of $7 \mathrm{~T}, 9.4 \mathrm{~T}, 10.5 \mathrm{~T}, 11.7 \mathrm{~T}$ and higher to benefit from increased SNR (Duyn 2012, Ladd et al. 2018), therefore richness of information, we are faced with increased $B_{0}$ field inhomogeneity (Juchem \& de Graaf 2017), causing, if not properly mitigated, severe image artifacts, notably geometric distortion in Echo Planar Imaging (EPI) sequences (Jezzard \& Balaban 1995). Spectroscopy is also largely impacted since inhomogeneous $B_{0}$ within a voxel translates into loss in spectral resolution. 
The magnetic field distribution $\Delta B_{0}(\boldsymbol{x})$ generated on top of the main $B_{0}$ field due to non-homogeneous media (considering non-ferromagnetic media), of magnetic susceptibility $\chi(\boldsymbol{x})$, can be expressed as (adapted from Salomir et al. (2003)):

$$
\nabla^{2}\left(\Delta B_{0}(\boldsymbol{x})\right)=\left(\nabla^{2} \chi(\boldsymbol{x})-3 \frac{\partial^{2} \chi(\boldsymbol{x})}{\partial z^{2}}\right) \frac{B_{0}}{3}
$$

Hence, the magnetic field inhomogeneity inside the human brain, apart from main field's intrinsic inhomogeneity, appears mainly due to magnetic susceptibility gradients between organic tissues and air cavities in the head. The intense $B_{0}$ field applied at their interface engenders non-uniform magnetization $\boldsymbol{M}(\boldsymbol{x})$ close to the brain, which in turn acts as a source of magnetic field characterized by a bounded current $\boldsymbol{J}_{b}(\boldsymbol{x})=\boldsymbol{\nabla} \times \boldsymbol{M}(\boldsymbol{x})$ generating the inhomogeneous field distribution.

Global inhomogeneity in a specific anatomy is commonly measured as the standard deviation $\sigma\left(\gamma \Delta B_{0}\right)$ (with $\gamma$ the gyro-magnetic ratio for the ${ }^{1} \mathrm{H}$ proton in $\mathrm{Hz} \mathrm{T}^{-1}$ ) computed from the sampled magnetic field excursion over all $K$ voxels of interest, namely those in the human brain in this study.

From Wald (2012), the geometric distortion in the Phase Encoding direction of an EPI sequence for a voxel with excursion $\Delta B_{0}$ is

$$
d_{P E}=\gamma \Delta B_{0} \tau_{E S} F O V_{P E}
$$

where, $\tau_{E S}$ is the echo-spacing time and $F O V_{P E}$ the Field of View in the phase encoding direction. At $\tau_{E S}=0.5 \mathrm{~ms}, F O V_{P E}=200 \mathrm{~mm}$ and $\gamma \Delta B_{0}=100 \mathrm{~Hz}$, geometric distortion computed from (2) would be $1.0 \mathrm{~cm}$, which is quite large for high resolution images. Moreover, voxels with excursions higher than $100 \mathrm{~Hz}$ are predominant at $7 \mathrm{~T}$ specially in the frontal and temporal lobes, thus making the use of homogenizing systems necessary.

The efforts to homogenize, or shim, the $B_{0}$ field occurs in steps spanning from magnet design to patient specific corrections using dedicated coils driven by electric currents calculated for each patient.

Since any magnetic field within a spherical region free of magnetic field sources can be fully described by Solid Spherical Harmonics (SH) (Roméo \& Hoult 1984, Chmurny \& Hoult 1990), MRI systems are equipped with a set of so called SH shim coils to generate SH shaped fields and counteract $\Delta B_{0}(\boldsymbol{x})$ harmonic orders greater than zero. Then a calibration step just before MRI scanning is performed, where a fieldmap of the anatomy of interest is acquired, $\mathrm{SH}$ coefficients are computed, and electric currents are injected into the coils to generate the adequate counteracting fields. Gradient coils perform the role of $1^{\text {st }}$ order shim concomitantly with their dynamic space-encoding task. In addition, most UHF MRI systems present $\mathrm{SH}$ shim coils at $2^{\text {nd }}$ and sometimes even $3^{\text {rd }}$ order, totaling 5 to 12 coils dedicated to SH shimming.

For shimming of the human brain at UHF, built-in shim coils present in most MRI systems are insufficient to eliminate $B_{0}$-related artifacts (Pan et al. 2012). Hence, there is a need for improving the shim system.

While the straightforward approach would be to increase SH orders by building dedicated high-order shim inserts, as proposed in Pan et al. (2012), Punchard (2013) and Kim et al. (2017), the amount of coils needed as a function of the desired SH order $l$ is $N(l)=l^{2}+2 l$. As more coils are needed, manufacturing complexity of both the shim insert and the associated electronics increases.

As a somewhat simpler alternative to $\mathrm{SH}$ based shimming, Multi-Coil Arrays (MCA) have been proposed for human brain shimming. They are composed of an 
array of independently-driven circular coils positioned around the subject's head (Juchem et al. 2011, Aghaeifar et al. 2018). The concomitant use of RF receive loops to signal reception and $B_{0}$ shimming has also been employed, taking advantage of the existing loops in the RF coil for this new task (Han et al. 2013, Stockmann et al. 2013, Stockmann et al. 2016). Due to the many channels composing an MCA (up to 48 channels in reported experiments), the system is intrinsically versatile, i.e. used for global brain shimming but most useful for dynamic slice-by-slice shimming. The MCAs composed of 30 to 48 channels presented great performance when used in dynamic shimming and overall good performance in global shimming, comparable to $5^{\text {th }}$ and $6^{\text {th }}$ order SH inserts (Stockmann \& Wald 2018).

So far, none of the systems described herein captured in their conception the specific magnetic field patterns in the human brain. As significant similarity among human brain fieldmaps can be observed, it could be argued that the generality of SH fields (as they form a basis for magnetic fields will null laplacian) is unnecessary when analyzing only one anatomy, and a smaller basis of field patterns could be obtained. Likewise, for MCAs, the location of the coils in most systems presented in the literature is likely non-optimal, with some recent exceptions in (Aghaeifar et al. 2020) and (Meneses et al. 2019).

Principal Component Analysis (PCA) has been proved useful to extract a small set of field patterns that can represent the most common field distributions in the human brain fieldmaps (Adalsteinsson et al. 1999). Shim coils could then be designed based on those field patterns.

Inspired by such a possibility, we developed a novel approach (Meneses \& Amadon 2019b, Meneses \& Amadon 2019c) allowing improved performance on global shimming while keeping a small amount of total channels. In the hereby-presented approach, instead of applying PCA over 3D $\Delta B_{0}$ fieldmaps, requiring creation of a common mask that may be bigger than some brains, causing unknown specification on the borders of the brain mask, PCA, by means of Singular Value Decomposition (SVD), is applied over more tractable 2D subject-optimized current density Stream Functions (Peeren 2003) on a cylindrical geometry. The application of SVD on a representative collection of subject-optimized (SO) stream functions (SF) leads to a smaller set of SFs that are discretized into coils for composing a shim system.

We remark that similar approaches have been presented recently (Jia et al. 2020, Arango et al. 2019, Can et al. 2019). In Jia et al. (2020), fieldmaps are shimmed by unconstrained SH of some order $\left(5^{\text {th }}\right.$ or $\left.6^{\text {th }}\right)$, then SO-SFs are computed under power dissipation constraints, tuned to achieve a residual field equivalent to that obtained by the SH shimming. Therefore, an indirect minimization of power dissipation is performed. While it is a sound approach, the resulting SVD based shim system presents relatively high channel count: 12 and 24 reported for achieving performances comparable to $5^{\text {th }}$ and $6^{\text {th }}$ order SH system, respectively, and although it is argued that these channel counts are lower than that of competing multi-coil array systems, manufacturing of SVD based coils is likely to be more complex, and therefore keeping the channel count to an even lesser amount should be favoured. Alternatively, a single channel SVD based shim system was presented in Can et al. (2019), providing relatively high inhomogeneity reduction that could be improved if more degrees of freedom were added.

In this paper, we give a detailed account of our SVD based coil design approach, and apply it on a database composed of 100 fieldmaps. It will be shown that a very reduced channel count system can be obtained by allowing power increase up to an 
optimal point.

\section{Methods}

The generation of a set of shim coils based on the actual magnetic field in the anatomy of interest consists mainly in three steps: (1) the acquisition of a representative database of fieldmaps $\Delta B_{0}^{s}(\boldsymbol{x})$ with $s=1, \ldots, S, S$ being the total number of subjects; (2) the computation of a SO-SF for each subject; and (3) the application of Singular Value Decomposition across the SO-SFs to obtain a reduced subset of SFs, subsequently discretized into windings at increasing cylinder radii.

\subsection{Dipole Boundary Method for Field-map Based Coil Design}

For SF computation, inspired by Inverse Boundary Element Methods (IBEM) (Pissanetzky 1992, Peeren 2003, Poole \& Bowtell 2007, Bringout \& Buzug 2015) and considering cylindrical geometry usually adopted for shim coil design, a simple inverse method is proposed to compute the SO-SFs. In this approach, a cylindrical surface is discretized into square loop elements, which may be considered as elementary magnetic dipoles, and the dipole current loop distribution is identified as the SF, providing a straightforward relation between magnetic field and SF.

We start by defining a conductive surface denoted $\mathcal{S} \subset \mathbb{R}^{3}$, a non-conductive region $\mathcal{V} \subset \mathbb{R}^{3}$ and a target magnetic field in the $z$ direction inside $\mathcal{V}$ through an application $B_{z}: \mathcal{V} \rightarrow \mathbb{R}$.

For a target field $B_{z}(\boldsymbol{x})$, either a current density $\boldsymbol{j}: \mathcal{S} \rightarrow \mathbb{R}^{3}$ or its associated SF $\psi: \mathcal{S} \rightarrow \mathbb{R}$ needs to be computed in order to extract the wire patterns upon which a nominal current $\mathcal{I}$ must flow to generate $B_{z}$. From Peeren (2003), those quantities relate by:

$$
\boldsymbol{j}(\boldsymbol{x})=\boldsymbol{\nabla} \psi(\boldsymbol{x}) \times \hat{\boldsymbol{n}}(\boldsymbol{x})
$$

with $\hat{\boldsymbol{n}}(\boldsymbol{x})$ the unitary vector normal to the surface $\mathcal{S}$ at point $\boldsymbol{x}$. Among several methods for coil design, the computation of the SF is preferred, since the inverse problem is simplified from estimating a vector field $\boldsymbol{j}$ into the estimation of a scalar function $\psi$. To address this inverse problem, we developed the Dipole Boundary Method (DBM) (Meneses \& Amadon 2019a), presented in detail herein.

In magneto-statics, the continuity equation for the current density is $\boldsymbol{\nabla} \cdot \boldsymbol{j}(\boldsymbol{x})=0$. Since $\boldsymbol{j}(\boldsymbol{x})$ is divergence-less, it can be broken up into a network of small current loops (Jackson 2007).

Naturally, this property remains valid for a surface. Therefore, any surface current density $\boldsymbol{j}(\boldsymbol{x})$ over a conductive surface $\mathcal{S}$ can be represented by an equivalent distribution of infinitesimal square loops with currents $I(\boldsymbol{x})$ over $\mathcal{S}$ (cf. Fig. 1a). It can be shown that the $I$-loop current network is equivalent to the $\boldsymbol{j}$-surface current density when it satisfies (cf. supplemental information):

$$
\boldsymbol{j}(\boldsymbol{x})=\boldsymbol{\nabla} I(\boldsymbol{x}) \times \hat{\boldsymbol{n}}(\boldsymbol{x})
$$

Identifying (3) and (4), $I(\boldsymbol{x})$ is a SF for $\boldsymbol{j}(\boldsymbol{x})$. The SF takes on a physical meaning as the current carried by each square loop in the network, therefore it can be directly related to the magnetic field. By defining a finite grid with a limited number of elementary loops, a piece-wise constant basis function is chosen for the SF estimation. The $I(\boldsymbol{x})$-current loop distribution constitutes a stream function for the current density 


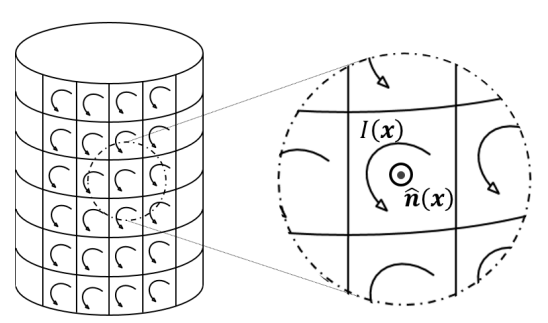

(a)

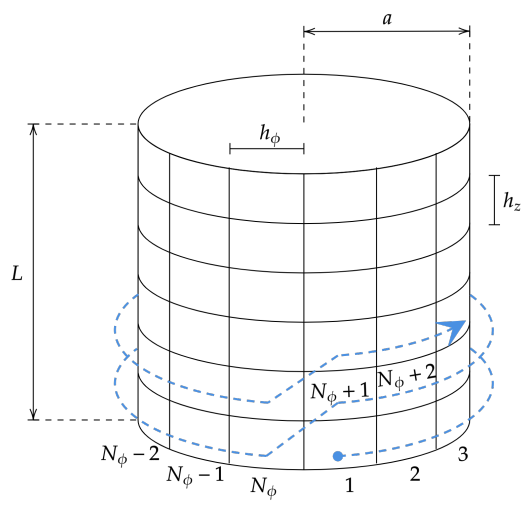

(b)

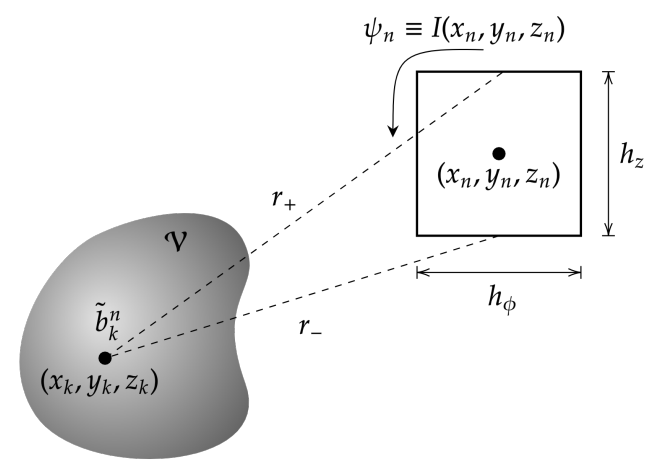

(c)

Figure 1: (a) Surface current density $\boldsymbol{j}(\boldsymbol{x})$ represented by a distribution of dipoles of current $I(\boldsymbol{x})$ over the cylindrical surface. $\hat{\boldsymbol{n}}(\boldsymbol{x})$ is the unitary normal vector at each point over the surface, (b) subsequent rectangular elements enumeration and (c) dipole with current $\psi_{n}$ generating a magnetic field $\tilde{b}_{k}^{n}$ in a control point of $\mathcal{V}$.

$\boldsymbol{j}$; i.e. the isoheight contours of $I(\boldsymbol{x})$ provide the current paths needed to approximate $j$ (Peeren 2003). There is no need to impose connectivity between the elementary current loop weightings, although power regularization will help obtain a smoother stream function, therefore less wire packing and less tortuous pathways.

From Biot-Savart law, the relation between the magnetic field $B_{z}(\boldsymbol{x})$ and the SF $\psi(\boldsymbol{x}) \equiv I(\boldsymbol{x})$ is easily obtained. The total magnetic field generated by the dipole network is simply the sum of the contributions of each dipole.

The coil former upon which the wires will be placed is defined as a cylinder of radius $a$ and length $L$.

$$
\mathcal{S}=\left\{(x, y, z) \mid x^{2}+y^{2}=a^{2},-\frac{L}{2} \leq z \leq \frac{L}{2}\right\}
$$


As the cylindrical surface is then discretized into square elements, let $h$ be the desired discretization step in $\phi$ and $z$; the number of elements in the azimuthal direction is $N_{\phi}=\lfloor 2 \pi a / h\rceil$ and $N_{z}=\lfloor L / h\rceil$ in the $z$ direction. As a consequence, effective discretization steps are $h_{\phi}=2 \pi a / N_{\phi}$ and $h_{z}=L / N_{z}$, generating rectangular elements which tend to squares as $h$ is reduced. Center coordinates $\boldsymbol{x}_{n}$ of dipole $n$ such as defined in Fig. 1b are:

$$
\boldsymbol{x}_{n}=\left(a \cos \left(\alpha \frac{h_{\phi}}{a}\right), a \sin \left(\alpha \frac{h_{\phi}}{a}\right),-\frac{L}{2}+\frac{2 \beta-1}{2} h_{z}\right)
$$

with $\alpha \equiv(n-1) \bmod N_{\phi}, \beta=(n-\alpha-1) / N_{\phi}+1$ and $n=1, \ldots, N$, with $N=N_{\phi} \times N_{z}$

Let the volume $\mathcal{V}$ be the anatomy of interest, the magnetic field excursion can be mapped over the $K$ voxels composing the discretized anatomy. The mapping is used as target field $\boldsymbol{b} \in \mathbb{R}^{K}$, of elements $b_{k}=\Delta B_{0}\left(x_{k}, y_{k}, z_{k}\right)$ where $k=1, \ldots, K$. BiotSavart law is then applied to compute the contribution $\tilde{b}_{k}^{n}$ of dipole $n$ with current $\psi_{n} \equiv I\left(x_{n}, y_{n}, z_{n}\right)$ to the total magnetic field in $z$ at point $\left(x_{k}, y_{k}, z_{k}\right)$. In the limit where $h \ll r_{ \pm}$the magnitude of the magnetic field is approximated by:

$$
\tilde{b}_{k}^{n}=c_{k, n} \psi_{n}
$$

with

$$
c_{k, n}=\left[\frac{\mu_{0}}{4 \pi a}\left(x_{n} x_{k}+y_{n} y_{k}-a^{2}\right)\left(\frac{1}{r_{-}^{3}}-\frac{1}{r_{+}^{3}}\right)\right] h_{\phi}
$$

and

$$
r_{ \pm}=\sqrt{\left(x_{k}-x_{n}\right)^{2}+\left(y_{k}-y_{n}\right)^{2}+\left[z_{k}-\left(z_{n} \pm \frac{h_{z}}{2}\right)\right]^{2}}
$$

The total magnetic field in $\boldsymbol{x}_{k}$ generated by the current distribution is

$$
\tilde{b}_{k}=\sum_{n=1}^{N} c_{k, n} \psi_{n}
$$

Finally, in a matrix/vector form:

$$
\tilde{b}=\mathbf{C} \boldsymbol{\psi},
$$

with $\mathbf{C}=\left(c_{k, n}\right) \in \mathbb{R}^{K, N}, \boldsymbol{\psi} \in \mathbb{R}^{N}$ and $\tilde{\boldsymbol{b}} \in \mathbb{R}^{K}$. The quadratic error between the target field and the coil field

$$
\varepsilon^{2}=|\boldsymbol{b}-\tilde{\boldsymbol{b}}|^{2}=|\boldsymbol{b}-\mathbf{C} \boldsymbol{\psi}|^{2}
$$

needs to be minimized by an appropriate choice of $\boldsymbol{\psi}$.

In addition to the quadratic error between target and produced magnetic field, power dissipation minimization needs to be taken into account for obtaining a feasible coil design. Mathematically, consideration of physical parameters of the coil such as power dissipation and stored magnetic energy acts as regularization for an otherwise ill-posed problem.

Inside any conducting region $\mathcal{V}_{c}$ with current density $\boldsymbol{J}(\boldsymbol{x})$ and electric conductivity $\kappa$, power dissipation can be calculated by: 


$$
P=\int_{\mathcal{V}_{c}} \frac{|\boldsymbol{J}(\boldsymbol{x})|^{2}}{\kappa} d v
$$

If the region is a homogeneous thin sheet of thickness $t$,

$$
P=\frac{1}{t \kappa} \int_{\mathcal{S}}|\nabla \psi(\boldsymbol{x}) \times \hat{\boldsymbol{n}}(\boldsymbol{x})|^{2} d s .
$$

For a cylindrical coil, the integral becomes:

$$
P=\frac{1}{t \kappa} \int_{\mathcal{S}}\left[\left(\frac{1}{a} \frac{\partial \psi}{\partial \phi}\right)^{2}+\left(\frac{\partial \psi}{\partial z}\right)^{2}\right] d s,
$$

which is discretized into:

$$
P \approx \frac{h_{\phi} h_{z}}{t \kappa} \sum_{n=1}^{N}\left[\left(\frac{\psi_{n+1}-\psi_{n}}{h_{\phi}}\right)^{2}+\left(\frac{\psi_{n+N_{\phi}}-\psi_{n}}{h_{z}}\right)^{2}\right] .
$$

With a discretization performed to make the elements as close as possible to squares $\left(h_{z} \approx h_{\phi}\right)$ :

$$
P \approx \frac{1}{t \kappa} \sum_{n=1}^{N}\left[\left(\psi_{n+1}-\psi_{n}\right)^{2}+\left(\psi_{n+N_{\phi}}-\psi_{n}\right)^{2}\right]
$$

or

$$
P \approx \boldsymbol{\psi}^{T} \mathbf{R} \psi
$$

where $\mathbf{R} \in \mathbb{R}^{N \times N}$ is a block Toeplitz matrix

$$
\mathbf{R}=\frac{2}{\kappa t}\left[\begin{array}{ccccc}
\mathbf{W} & -\mathbf{I} & \mathbf{0} & \ldots & \mathbf{0} \\
-\mathbf{I} & \mathbf{W} & -\mathbf{I} & \ldots & \mathbf{0} \\
\mathbf{0} & -\mathbf{I} & \mathbf{W} & \ldots & \mathbf{0} \\
\vdots & \vdots & \vdots & \ddots & \vdots \\
\mathbf{0} & \mathbf{0} & \mathbf{0} & \ldots & \mathbf{W}
\end{array}\right]
$$

with $\mathbf{W} \in \mathbb{R}^{N_{\phi} \times N_{\phi}}$ a circulant matrix of first column $\boldsymbol{w}=\left[\begin{array}{llllll}4 & -1 & 0 & \ldots & 0 & -1\end{array}\right]^{T}$, $\mathbf{I} \in \mathbb{R}^{N_{\phi} \times N_{\phi}}$ an identity matrix and $\mathbf{0} \in \mathbb{R}^{N_{\phi} \times N_{\phi}}$ a null matrix.

To impose that no current flows outwards or inwards of the cylinder from its top or bottom, the SF value on each of these boundaries must be constant (Pissanetzky 1992, Peeren 2003, Poole \& Bowtell 2007, Bringout \& Buzug 2015). This forces the first $N_{\phi}$ elements in $\boldsymbol{\psi}$ to be of equal unknown value $\boldsymbol{\psi}_{1}^{\prime}$; and also forces the last $N_{\phi}$ elements of $\boldsymbol{\psi}$ to have equal unknown values $\boldsymbol{\psi}_{N^{\prime}}^{\prime}$. This imposition is expressed by the computation of a reduced SF $\boldsymbol{\psi}^{\prime}$ with $N^{\prime}=N-2\left(N_{\phi}-1\right)$ elements such that $\psi_{i}^{\prime}=\psi_{i+N_{\phi}-1}$. The boundary-conditioned SF $\boldsymbol{\psi}^{\prime}$ relates to $\boldsymbol{\psi}$ through the matrix formulation: $\boldsymbol{\psi}=\boldsymbol{\Gamma} \boldsymbol{\psi}^{\prime}$ with $\boldsymbol{\Gamma} \in \mathbb{R}^{N \times N^{\prime}}$ of form:

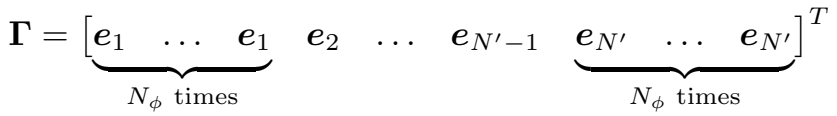

where $\left\{\boldsymbol{e}_{i}\right\}$ is the standard basis for $\mathbb{R}^{N^{\prime}}$, e.g. $\boldsymbol{e}_{1}=\left[\begin{array}{llll}1 & 0 & \ldots & 0\end{array}\right]^{T}$. 
Assembling power consumption and quadratic field error, optimal $\boldsymbol{\psi}$ is

$$
\boldsymbol{\psi}(\lambda)=\underset{\boldsymbol{\psi}^{\prime} \in \mathbb{R}^{N^{\prime}}}{\operatorname{argmin}} \lambda \boldsymbol{\psi}^{\prime T} \boldsymbol{\Gamma}^{T} \mathbf{R} \boldsymbol{\Gamma} \boldsymbol{\psi}^{\prime}+\left\|\boldsymbol{b}-\mathbf{C} \boldsymbol{\Gamma} \boldsymbol{\psi}^{\prime}\right\|_{2}^{2}
$$

where $\lambda$ is a regularization parameter that can be tuned to balance the solution in terms of reducing power dissipation or increasing magnetic field fidelity.

The functional is then minimized by:

$$
\boldsymbol{\psi}(\lambda)=\boldsymbol{\Gamma D} \boldsymbol{\Gamma}^{T} \mathbf{C}^{T} \boldsymbol{b}
$$

with

$$
\mathbf{D}=\left[\boldsymbol{\Gamma}^{T}\left(\lambda \mathbf{R}+\mathbf{C}^{T} \mathbf{C}\right) \boldsymbol{\Gamma}\right]^{-1} .
$$

We note that any coil design method could be used in this step. Nevertheless, the method presented herein does not require a third-party triangular mesher, contrarily to conventional IBEM, and the adoption of a piecewise constant basis function for the SF avoids the computation of derivatives of the SF within each element and subsequent integration over the elemental surface. The contribution of each element to the magnetic field is restricted to the edges of the squares and is easily calculated. On the other hand, accuracy of this approximation will depend on mesh resolution, and very high resolution may be a computational burden. Appropriate choice of $h$ will depend on the coil radius, and on the extent of the target field domain.

The SF is then used to obtain the geometric center of the wires that must be placed over the coil former so that a magnetic field of high fidelity to the target is generated.

The process of discretization into windings is performed by fixing a minimum allowed distance $\delta_{w}$ between any two wire's geometric centers. This distance bounds the maximum section $w_{s}$ of circular wire that can be employed on the actual manufacturing of the coil and will also dictate the current $\mathcal{I}$ that needs to flow in the wires to generate the magnetic field that would be generated by $\boldsymbol{j}(\boldsymbol{x})=\boldsymbol{\nabla} \psi(\boldsymbol{x}) \times \hat{\boldsymbol{n}}(\boldsymbol{x})$. Smaller $\delta_{w}$ provides higher magnetic field fidelity with the field that would be generated by the continuous current distribution $\boldsymbol{j}(\boldsymbol{x})$. On the other hand, for the relatively complex patterns that will be presented, low $\delta_{w}$ might make manufacturing difficult. In addition, power dissipation would increase.

From a nominal current $\mathcal{I}$, a family of isoheight curves of $\psi$ representing the geometric centers of the coil wires can be obtained as detailed in (Peeren 2003). Since $\delta_{w}$ is imposed, the nominal current needs to be calculated such that the resulting discretization into windings does not violate this supremum, a condition satisfied by:

$$
\begin{aligned}
\mathcal{I} & =\max _{\boldsymbol{x} \in \mathcal{S}}|\boldsymbol{j}(\boldsymbol{x})| \delta_{w} \\
& =\max _{\boldsymbol{x} \in \mathcal{S}}|\boldsymbol{\nabla} \psi(\boldsymbol{x})| \delta_{w} .
\end{aligned}
$$

Having obtained the family of loops that compose the coil for a given $\delta_{w}$, let $L_{c}$ with $c=1, \ldots, C$ be the length of each of a total of $C$ loops; the resistance of the coil can be calculated by:

$$
r=\frac{1}{\sigma w_{s}} \sum_{c=1}^{C} L_{c}
$$


which in turn is used to calculate the coil's power dissipation:

$$
\mathcal{P}=r \mathcal{I}^{2}
$$

At nominal current, the inhomogeneity of the shimmed fieldmap is the standard deviation of the residual field: $\sigma(\boldsymbol{b}-\mathbf{C} \boldsymbol{\psi})$. For purposes of performance evaluation, we define a metric $\eta$ for inhomogeneity reduction (percentage rate):

$$
\eta=100 \times\left(1-\frac{\sigma(\boldsymbol{b}-\mathbf{C} \boldsymbol{\psi})}{\sigma(\boldsymbol{b})}\right)
$$

Finally, different values of $\lambda$ will provide different values of $\mathcal{P}$ and $\eta$. Decreasing $\lambda$ allows inhomogeneity reduction to increase (by reducing the quadratic error $\varepsilon^{2}$ ), at the cost of power dissipation increase. Hence $\lambda$ needs to be tuned to obtain an appropriate trade-off. In the subsequent group analysis, for each subject in the database, the regularization parameter is tuned such that the resulting subject-optimal coil dissipates some desired target power $\mathcal{P}_{T}$; this process is equivalent to solving the inhomogeneity minimization under power constraints.

\subsection{Singular Value Decomposition of Optimal SFs}

For a fixed coil former geometry and individual regularization parameter $\lambda_{s}$, each subject's offset map $\boldsymbol{b}_{s}=\left[\begin{array}{lllll}\Delta B_{0}^{s}\left(\boldsymbol{x}_{1}\right) & \Delta B_{0}^{s}\left(\boldsymbol{x}_{2}\right) & \ldots & \Delta B_{0}^{s}\left(\boldsymbol{x}_{K_{s}}\right)\end{array}\right]^{T}$ is input as target field in the DBM algorithm, outputting a SO-SF $\psi_{s}$.

From the resulting set of $S$ SO-SFs $\boldsymbol{\psi}_{s}$ calculated from a representative database of $S$ subjects, the goal is to obtain a reduced set of $M(<S)$ new SFs that could approximate the effects of each SO-SF, within a certain error, by adjusting the coefficients of their linear combination. To do so, Singular Value Decomposition is applied.

The SFs calculated for each target field in the database are assembled into a matrix $\boldsymbol{\Psi}_{\mathrm{DB}}=\left[\begin{array}{llll}\boldsymbol{\psi}_{1} & \boldsymbol{\psi}_{2} & \ldots & \boldsymbol{\psi}_{S}\end{array}\right] \in \mathbb{R}^{N \times S}$. The matrix $\boldsymbol{\Psi}_{\mathrm{DB}}$ possesses an SVD, expressed as:

$$
\mathbf{\Psi}_{\mathrm{DB}}=\mathbf{U} \boldsymbol{\Sigma} \mathbf{V}^{T}
$$

where $\mathbf{U} \in \mathbb{R}^{N \times N}$ and $\mathbf{V} \in \mathbb{R}^{S \times S}$ are orthogonal matrices whose columns are eigenvectors of $\boldsymbol{\Psi}_{\mathrm{DB}} \boldsymbol{\Psi}_{\mathrm{DB}}^{T}$ and $\boldsymbol{\Psi}_{\mathrm{DB}}^{T} \boldsymbol{\Psi}_{\mathrm{DB}}$ respectively, and $\boldsymbol{\Sigma} \in \mathbb{R}^{N \times S}$ is a diagonal matrix of singular values of $\boldsymbol{\Psi}_{\mathrm{DB}}$.

From the SVD, we define the matrix $\boldsymbol{\Psi}_{\mathrm{SVD}}$ as:

$$
\mathbf{\Psi}_{\mathrm{SVD}}=\mathbf{U} \boldsymbol{\Sigma}
$$

where $\boldsymbol{\Psi}_{\mathrm{SVD}}=\left[\begin{array}{llll}\boldsymbol{\psi}_{1}^{\mathrm{SVD}} & \boldsymbol{\psi}_{2}^{\mathrm{SVD}} & \ldots & \boldsymbol{\psi}_{S}^{\mathrm{SVD}}\end{array}\right] \in \mathbb{R}^{N \times S}$ is a matrix whose columns are a new set of SFs that by appropriate choice of linear coefficients, can be combined to reconstruct $\boldsymbol{\Psi}_{\mathrm{DB}}$.

The SVD modes represented by the columns of $\boldsymbol{\Psi}_{\mathrm{SVD}}$ are ordered in the matrix such that the first column is the mode that presents the highest correlation to the whole set of SO SFs, the second column is the second most correlated and so on. The elements in the diagonal of $\boldsymbol{\Sigma}$ are then the $\boldsymbol{\Psi}_{\mathrm{DB}}$ singular values in decreasing order; they are a measure of pertinence of each SVD mode in the reconstitution of $\boldsymbol{\Psi}_{\mathrm{DB}}$.

If all SVD modes are used for the reconstruction of $\boldsymbol{\Psi}_{\mathrm{DB}}$ the linear coefficients for the reconstruction of each column are in the columns of $\mathbf{V}^{T}$, such that: 


$$
\boldsymbol{\Psi}_{\mathrm{DB}}=\boldsymbol{\Psi}_{\mathrm{SVD}} \mathbf{V}^{T}
$$

Since we intend to obtain a reduced dimension subset of SFs, only the first few columns of $\boldsymbol{\Psi}_{\mathrm{SVD}}$ are retained to constitute the shim system.

Retaining the $M$ first columns of $\boldsymbol{\Psi}_{\mathrm{SVD}}$, the computation of performance and power consumption of a physical realization of the shim system starts with the discretization into windings of $\boldsymbol{\psi}_{m}^{\mathrm{SVD}}$ for $m=1, \ldots, M$. From this discretization, the resistance $r_{m}$ and nominal current $\mathcal{I}_{m}$ of each SF-SVD coil is calculated.

For a fieldmap $\boldsymbol{b}$ being shimmed by the SF-SVD system, the optimal currents $\boldsymbol{i}=\left[\begin{array}{llll}i_{1} & i_{2} & \ldots & i_{M}\end{array}\right]$ to be injected in each coil are calculated such that their application minimizes the quadratic error between target field and coil generated field:

$$
\boldsymbol{i}=\underset{\boldsymbol{i} \in \mathbb{R}^{M}}{\operatorname{argmin}}\left\|\boldsymbol{b}-\mathbf{C} \sum_{m=1}^{M} i_{m} \frac{\boldsymbol{\psi}_{m}^{\mathrm{SVD}}}{\mathcal{I}_{m}}\right\|_{2}^{2} .
$$

Total power dissipation and performance in inhomogeneity reduction for a shimmed subject are then calculated as:

$$
\mathcal{P}_{\mathrm{SVD}}=\sum_{m=1}^{M} r_{m} i_{m}^{2}
$$

and

$$
\eta_{\mathrm{SVD}}=100 \times\left(1-\frac{\sigma\left(\boldsymbol{b}-\mathbf{C} \sum_{m=1}^{M} i_{m} \frac{\boldsymbol{\psi}_{m}^{\mathrm{SVD}}}{\mathcal{I}_{m}}\right)}{\sigma(\boldsymbol{b})}\right) .
$$

\subsection{SVD Coil Calculation over Multiple Radii}

The SF-SVD coil generation method described so far produces a number of different coils over the exact same cylindrical surface for all modes. This is not possible for a real system fabrication, thus the passage of SVD modes $m>1$ to cylinders of larger radii $a_{m}>a$ is necessary. These new radii are defined according to the space necessary to accommodate the wires, together with the supporting structure upon where the wires will be placed.

Departing from $\boldsymbol{\psi}_{m}^{\mathrm{SVD}}$ over a cylindrical surface of radius $a$, a new SF $\boldsymbol{\psi}_{m, a_{m}}^{\mathrm{SVD}}$ over a cylinder of radius $a_{m}$ such that both coils produce the same magnetic field in a region of interest $\mathcal{V}$ needs to be calculated.

The region of interest $\mathcal{V}$ should enclose all brains in the fieldmap database. The simplest region for the task is a sphere centered at the isocenter. Let $\mathcal{X}_{s}$ be the set of coordinates $\boldsymbol{x}_{k}$ of the $K_{s}$ voxels of subject $s$, the radius of the smallest sphere enclosing all brains is $R_{\mathrm{sph}}=\sup \mathcal{R}$, where $\mathcal{R}=\left\{\|\boldsymbol{x}\|_{2} \mid \boldsymbol{x} \in \cup_{s=1}^{S} \mathcal{X}_{s}\right\}$.

Since the magnetic field generated by any external coil obeys Laplace's equation inside $\mathcal{V}$, the magnetic field over the boundary $\partial \mathcal{V}$ of $\mathcal{V}$, a spherical surface, is sufficient as target field for the computation of the new SF. Performing a regular discretization of the spherical surface into $K_{\mathrm{sph}}$ points, the matrices $\mathbf{C}_{\mathrm{sph}}$ and $\mathbf{C}_{\mathrm{sph}, a_{m}}$ associating, respectively, $\boldsymbol{\psi}_{m}^{\mathrm{SVD}}$ and $\boldsymbol{\psi}_{m, a_{m}}^{\mathrm{SVD}}$ to the magnetic field they generate over $\partial \mathcal{V}$ can be 
computed and the new equivalent $\mathrm{SF}$ on the greater radius is:

$$
\boldsymbol{\psi}_{m, a_{m}}^{\mathrm{SVD}}=\boldsymbol{\Gamma}_{a_{m}} \mathbf{D}_{\mathrm{sph}, a_{m}} \boldsymbol{\Gamma}_{a_{m}}^{T} \mathbf{C}_{\mathrm{sph}, a_{m}}^{T} \mathbf{C}_{\mathrm{sph}} \frac{\boldsymbol{\psi}_{m}^{\mathrm{SVD}}}{\mathcal{I}_{m}} .
$$

with

$$
\mathbf{D}_{\mathrm{sph}, a_{m}}=\left[\boldsymbol{\Gamma}_{a_{m}}^{T}\left(\lambda \mathbf{R}_{a_{m}}+\mathbf{C}_{\mathrm{sph}, a_{m}}^{T} \mathbf{C}_{\mathrm{sph}, a_{m}}\right) \boldsymbol{\Gamma}_{a_{m}}\right]^{-1} .
$$

The regularization parameter $\lambda$ must be tuned to guarantee that the newly generated SF will produce a magnetic field to maintain the same shim system performance with minor or no increase in total power consumption.

Finally, after adequate "projections" of the SF-SVD coils onto larger cylindrical surfaces, a physically realizable set of shim coils is obtained.

\subsection{Constitution of a Brain Fieldmap Database}

A database of brain fieldmaps was assembled from MRI acquisitions of 100 consenting and healthy adult subjects, consisting of a $53 / 47$ male to female ratio, of average 60 years (SD: 10$)$ and $70 \mathrm{~kg}$ (SD: 15 ). The $\Delta B_{0}$ brain maps were obtained at $3 \mathrm{~T}$ from a Prisma scanner (Siemens Healthcare, Erlangen) equipped with a 20-channel RF head coil and full $2^{\text {nd }}$ order $\mathrm{SH}$ shim coils.

For accurate $\Delta B_{0}$ estimation, we based our $\Delta B_{0}$ mapping on 3 echoes rather than 2. Yet to avoid sometimes tedious spatial phase unwrapping, we opted for temporal phase unwrapping of the third echo, based on the assumption that no phase excursion occurs between the first and second echoes beyond $\pm \pi$. This means that the first 2 echoes must be placed extremely close to one-another $(0.7 \mathrm{~ms}$ to catch $B_{0}$ excursions within $\pm 714 \mathrm{~Hz}$ ), which cannot be reached in a single sequence. Therefore after $2^{\text {nd }}$ order $\mathrm{SH}$ shimming, a $3 \mathrm{D}$ gradient echo sequence was played twice, one with 2 distant echoes $T E_{1}=1.88 \mathrm{~ms}$ and $T E_{3}=4.9 \mathrm{~ms}$ ), and one with a single echo at $T E_{2}=T E_{1}+0.7 \mathrm{~ms}$. Then a triple-point linear fit of the phase evolution was performed by gathering all three echoes, with correction of the potential temporal phase-unwrapping of the third echo based on the slope given by the first two echoes. The other sequence parameters were: sagittal orientation, isotropic voxel resolution $=1.7 \mathrm{~mm}, T R=10 \mathrm{~ms}$, Flip Angle $=8^{\circ}, 2 \mathrm{D}$ Caipirinha acceleration factors $=2 \times 2, T A=44 \mathrm{~s}$. The resulting $\Delta B_{0}$ maps were cleaned with an outlier filter to avoid singularities, especially at the edge of the brain; the filter marked a brain voxel as outlier by comparing its excursion from the median to the variance, both statistics estimated from its neighbors; such outlier values were then replaced with their neighboring median. A mask of the brain was extracted from the magnitude image using FSL's Brain Extraction Tool. The quality of the brain masks and fieldmaps was checked visually in at least the three orthogonal central slices for each subject. Since the magnetization $\boldsymbol{M}$ of tissues generating the inhomogeneous magnetic field is $\boldsymbol{M}=\left(\chi / \mu_{0}\right) B_{0} \hat{\boldsymbol{z}}$, the $\Delta B_{0}$ fieldmaps were linearly re-scaled to represent the inhomogeneity corresponding to a $7 \mathrm{~T}$ main field with no loss of accuracy. The acquisition at lower fields is advantageous as field inhomogeneity is lower, thus reducing geometric distortion and signal loss compared to what would be obtained at $7 \mathrm{~T}$. The average and standard deviation of the inhomogeneity of the entire fieldmap database is $\bar{\sigma}_{\text {base }}=65.7 \mathrm{~Hz}(11.4)$ after re-scaling to $7 \mathrm{~T}$. 


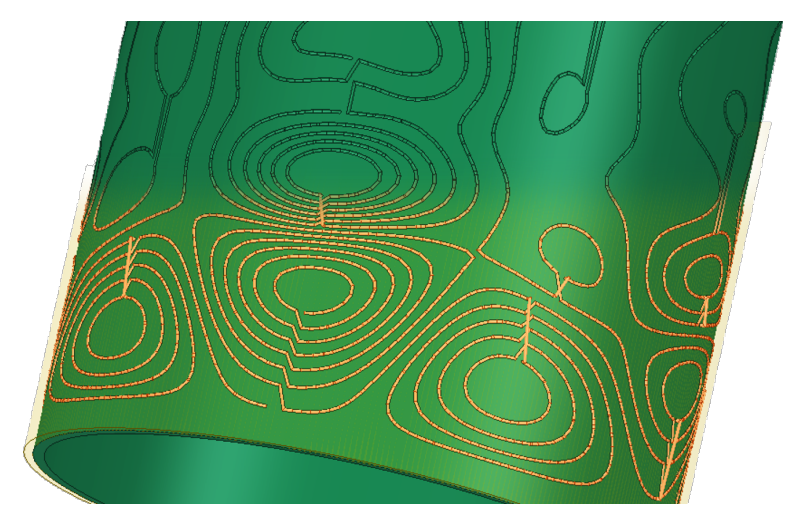

Figure 2: 3D model of a realistic system setup for a single layer (channel) of an SF-SVD shim system; depicting groove paths for wire accommodation (top) and realistic wiring of an SVD coil layer (bottom). Note the wire bridges in the dimension orthogonal to the cylindrical surface to connect concentric loops.

\subsection{Choice of Design and Validation Parameters}

Our goal is to achieve optimal shimming with a low power budget. Limiting power dissipation helps reduce costs related to both electronics and heat management. By guaranteeing low power dissipation, dedicated cooling systems can be avoided, as the forced air flow in the MRI tunnel may be sufficient to maintain a low, safe temperature. The most obvious way to limit the required power is to make the cylindrical shim set as close as possible to the human head. Thus, the cylindrical coil former dimensions were set to $a=140 \mathrm{~mm}$ (so that it could be placed at the exterior of our in-house $\mathrm{RF}$ head coil) and $L=300 \mathrm{~mm}$. Mesh resolution for SF computation is set to $h=4 \mathrm{~mm}$. To assess accuracy of the piecewise constant basis function under this particular discretization, different values of $h$ were tested for one random fieldmap in the database, with insignificant changes in performance and power consumption for $h$ inferior to $4 \mathrm{~mm}$.

Discretization into windings for SO and SVD coils is performed with $\delta_{w}=2.4 \mathrm{~mm}$ and with copper wire of $1.54 \mathrm{~mm}^{2}$ circular section $(1.4 \mathrm{~mm}$ diameter) and electric conductivity $\kappa=5.96 \times 10^{7} \mathrm{~S} \mathrm{~m}^{-1}$. Under this discretization, characteristic power dissipation of each coil and performance over its associated map are calculated from (26) and (27).

This particular choice of discretization parameters allows the use of relatively large copper wire gauge, resulting in lower power dissipation at high current. Large wire gauges tend to preserve their form once bent, a convenient characteristic for manufacturing. Moreover, the $2.4 \mathrm{~mm}$ inter-wire spacing leaves enough room for a manufacturing based on accommodating the wires into grooves milled onto a support structure, as depicted in Fig. 2.

For analysis of system performance at different power dissipation values, 8 power targets were chosen for SO-SF generation: $1 \mathrm{~W}, 3 \mathrm{~W}, 7 \mathrm{~W}, 15 \mathrm{~W}, 25 \mathrm{~W}, 50 \mathrm{~W}, 75 \mathrm{~W}$ and $100 \mathrm{~W}$. The regularization parameter for each subject is tuned to reach each power target. Hence, eight sets of SFs $\boldsymbol{\Psi}_{\mathrm{DB}}^{1 W}, \boldsymbol{\Psi}_{\mathrm{DB}}^{3 W}, \ldots, \boldsymbol{\Psi}_{\mathrm{DB}}^{100 W}$ are obtained and subsequently decomposed into the SVD SFs $\boldsymbol{\Psi}_{\mathrm{SVD}}^{1 W}, \mathbf{\Psi}_{\mathrm{SVD}}^{3 W}, \ldots, \boldsymbol{\Psi}_{\mathrm{SVD}}^{100 W}$. Note that in this step, average power across the SO coils is forced to be very close to the power 
target and with low standard deviation, but there is no guarantee that the resulting SF-SVD coils will present the same power dissipation characteristics. The superscript on $\boldsymbol{\Psi}_{\text {SVD }}^{\mathcal{P}_{T}}$ serves only to associate the SVD SF to its generating SO-SFs.

The shimming capabilities of the SF-SVD system over "new" fieldmaps is assessed by generating the SF-SVD coils from 50 randomly selected maps forming a design set $\mathcal{D}$ and simulating the system's performance over $\mathcal{D}$ and the set of remaining 50 fieldmaps $\mathcal{T}$, called test set. This is known as the hold-out method for validation. The sets $\mathcal{D}$ and $\mathcal{T}$ are kept the same for the eight power targets so as to provide comparable systems. Unless specified otherwise, the performance and power estimations will be reported for $\mathcal{T}$.

It will be observed that $\mathcal{P}_{T}=15 \mathrm{~W}$ provides a good compromise between inhomogeneity reduction and power dissipation. Therefore, the resulting $\Psi_{\mathrm{SVD}}^{15 \mathrm{~W}} \mathrm{SF}-$ SVD shim system will be retained for subsequent analysis on robustness of the method and comparison to SH shimming.

\subsection{Cross-validation}

For a more statistically significant evaluation of the SF-SVD method's robustness to new fieldmaps, 10 -fold cross-validation is performed for $15 \mathrm{~W}$ target power SO-SFs. In this analysis, the fieldmap database is divided into 10 disjoint clusters $\mathcal{K}_{j}, j=1, \ldots, 10$, composed by 10 fieldmaps each. Each cluster is then used once as test set $\mathcal{T}_{j}=\mathcal{K}_{j}$, with the remaining clusters used as design set $\mathcal{D}_{j}=\cup_{i \neq j} \mathcal{K}_{i}$. For each generation, average performance of the resulting SF-SVD shim system is evaluated over $\mathcal{D}_{j}$ and $\mathcal{T}_{j}$ and the resulting ratios $\bar{\eta}\left(\mathcal{T}_{j}\right) / \bar{\eta}\left(\mathcal{D}_{j}\right)$ are used as a metric to evaluate how well the SF-SVD system behaved over the new subjects. Only the first 3 SVD modes for each generation are retained to compose the SF-SVD system.

\subsection{Performance Assessment and Realistic Design Evaluation}

Comparison of the $\boldsymbol{\Psi}_{\text {SVD }}^{15 W}$ SF-SVD shim system performance against unlimited power high-order SH shimming is then carried-out.

To avoid computational burden, the simulations described so far are performed assuming the SVD coils are at the same cylindrical radius and magnetic fields over the ROIs are computed directly from the ideal stream functions. Nevertheless, to validate this approximation, we explore a feasible system design, whereby the projection of the $2^{\text {nd }}$ and $3^{\text {rd }} \boldsymbol{\Psi}_{\text {SVD }}^{15 W}$ modes is performed upon radii $a_{2}$ and $a_{3}$, chosen to be $144.8 \mathrm{~mm}$ and $149.6 \mathrm{~mm}$ respectively. This choice leaves a $4.8 \mathrm{~mm}$-distance between successive channel wiring centers, providing enough space for the return wires, resin coating for fixation and the associated supporting structure of each coil. The first SF-SVD coil is maintained unchanged over radius $a=140 \mathrm{~mm}$. The spherical surface considered for target field calculation has $12 \mathrm{~cm}$ radius and is discretized such that the amount of control points is in the order of the number of voxels in the ROIs of our database (roughly 300,000 voxels in the brain). The behavior of this new, feasible system is then evaluated. Afterwards, discretization into windings is performed on the projected stream functions and the magnetic field generated in the ROIs is now computed from the current flow in the actual current paths using Biot-Savart law. The eventual loss of performance of the realistic winding system is assessed.

Overall, the dimensions of a 3-layer shim insert would be the following: considering $5 \mathrm{~mm}$ thickness for inner and outer cylindrical formers including burried 
wires, a total thickness of about $2 \mathrm{~cm}$ would be expected, with $27 \mathrm{~cm}$ internal diameter and $30 \mathrm{~cm}$ length.

\section{Simulation Results and Discussion}

\subsection{Subject Optimal SF Computation}

After tuning of the regularization parameter and SF computation for the eight power targets, resulting mean and standard deviation of power dissipation across the $100 \mathrm{SO}$ coils are: $1.0 \mathrm{~W}(0.04), 3.1 \mathrm{~W}(0.1), 7.2 \mathrm{~W}(0.4), 15.4 \mathrm{~W}(0.9), 25.7 \mathrm{~W}(1.5), 51.9 \mathrm{~W}$ (3.3), $77.7 \mathrm{~W}(5.7)$ and $104.1 \mathrm{~W}(11.8)$, which are sufficiently close to the desired power dissipation targets for the purposes of this analysis.

Performance and final inhomogeneity obtained from SO-SF shimming are reported as functions of mean power dissipation in Fig. 3.
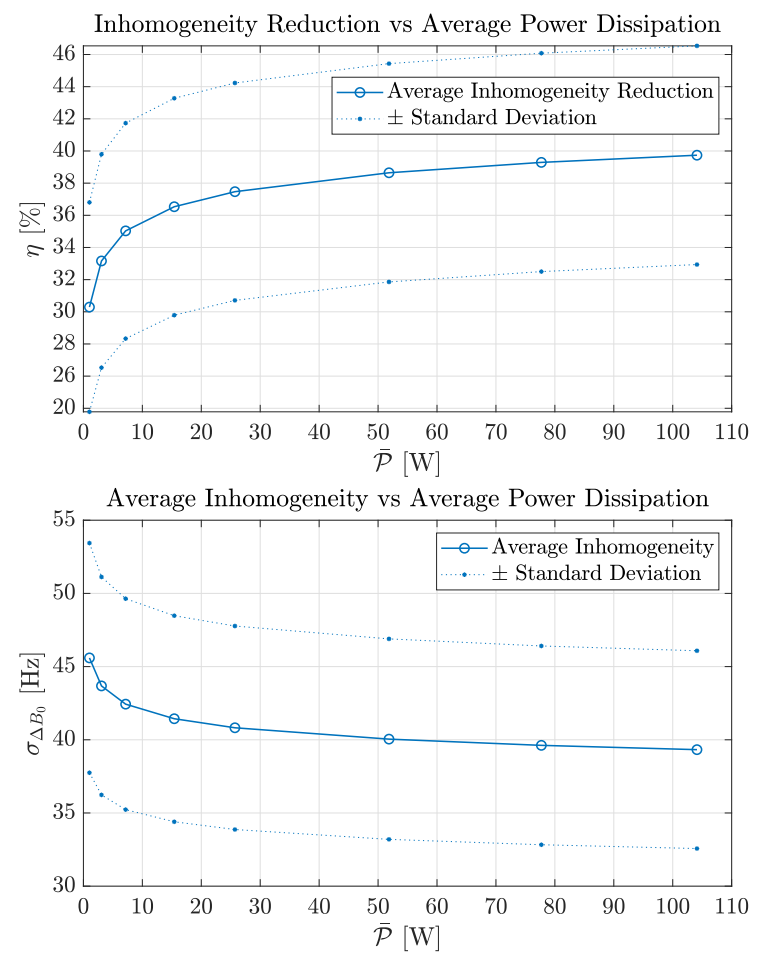

Figure 3: Inhomogeneity reduction and final inhomogeneity at $7 \mathrm{~T}$ over a 100 -subject database after SO coil shimming for increasing power dissipation designs.

As expected from the functional in (21), it can be observed that a steady reduction of B0 inhomogeneity requires more than an exponential power increase. Improvement in average performance from $\bar{\eta}=37.5 \%$ to $39.7 \%$ (from $\bar{\sigma}_{\Delta B_{0}}=40.8 \mathrm{~Hz}$ to $39.3 \mathrm{~Hz}$ ) demands $78.4 \mathrm{~W}$ average power increase. For a system close to the patient's head, such growth in power dissipation, initially at $25.7 \mathrm{~W}$, to obtain an absolute $2.2 \%$ gain in performance, does not seem justifiable. On the other hand, the lowest performance shown in Fig. 3, $30.3 \%\left(\bar{\sigma}_{\Delta B_{0}}=45.6 \mathrm{~Hz}\right)$, is already superior to a full $5^{\text {th }}$ order $\mathrm{SH}$ shimming (cf. Fig. 8), and the associated average power dissipation is merely $1.0 \mathrm{~W}$. 

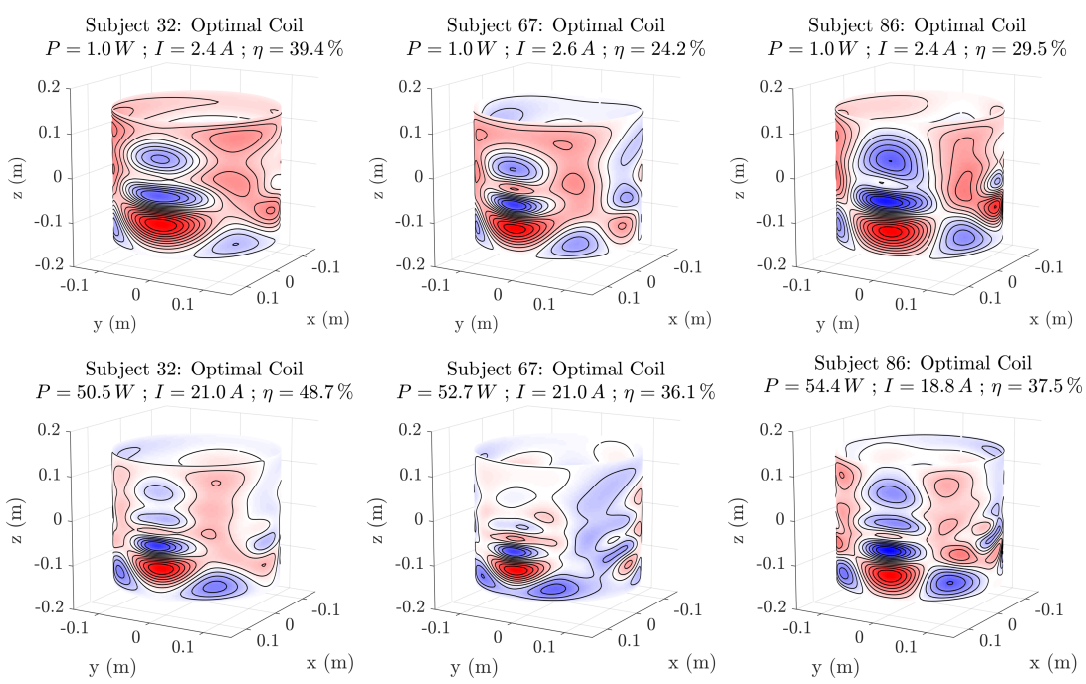

Figure 4: Wire geometric centers of subject-optimal coils for 3 subjects at two different performances and power dissipation for each subject. The colormap represent the SF intensity around the cylindrical surface (red is positive, blue is negative, which gives the sign of the current flow in the depicted windings).

A raise in power dissipation from $1.0 \mathrm{~W}$ to $25.7 \mathrm{~W}$ provides an absolute increase of $7.2 \%$ in average performance, showing a better performance increase to power increase ratio, and keeping the system under acceptable power dissipation levels. The greater improvement in performance within the lower power ranges is clearly marked, where it seems that power dissipation ranging from $15 \mathrm{~W}$ to $25 \mathrm{~W}$ should be privileged. Indeed, low power dissipation designs can be driven by low budget electronics and need not specific heat dissipation management.

Regarding the SO coils' wiring patterns in Fig 4, improvement of the brain magnetic field homogeneity requires the shape of the coil wirings to change and their nominal current and power to increase. In particular, the SF presents more rapid variations, leading to a more complex current flow needed to better address high magnetic field intensity and variations without degrading initially small magnetic field values. The circumference of current loops tends to decrease in this process, demanding higher current for generating a same magnetic field intensity.

The similarity between the wire patterns shown for these three subjects, also observed among all SO coils, is remarkable. There are concentrations of current flow in the front of the coil, to address the intense inhomogeneity in the orbito-frontal cortex. High current flow can also be observed in the regions close to the ears, which also present intense inhomogeneity caused by the interfaces with the ear canals. This similarity among coil patterns is an indicator that a small and effective shim coil system for the brain could be obtained through Singular Value Decomposition. 


\subsection{SF-SVD Coil Generation and Evaluation}

Singular value decomposition is applied on each of the eight subject-optimal SF sets. The SF-SVD coil designs are shown in Fig. 5, where a tendency for symmetry can be observed, especially on the first mode. These wiring patterns are consistent with the overall brain symmetry. As the power consumption of the shim system is allowed to increase to provide better performance, it is once again observed that the loops associated to the current paths become shorter in length.
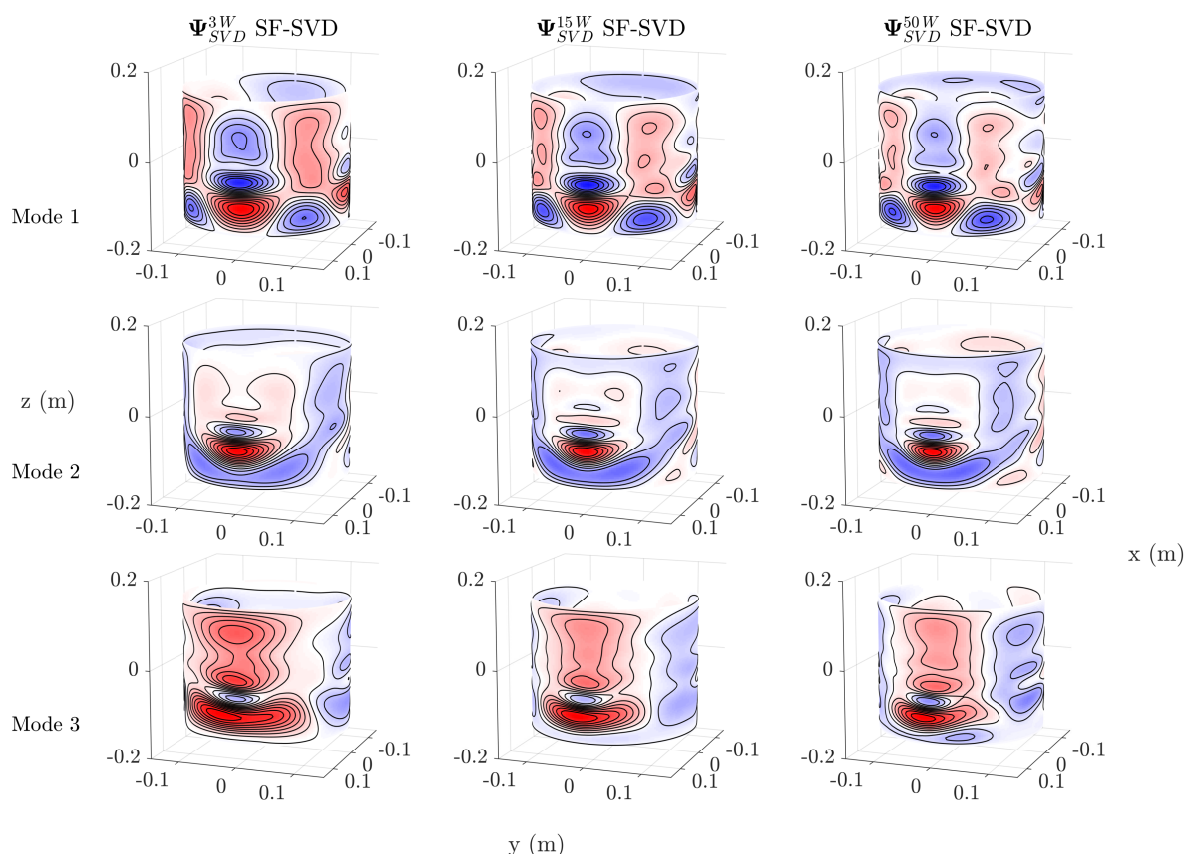

Figure 5: Geometric centers of the windings obtained from the first three SF-SVD modes with increasing power dissipation. The colormap indicates the intensity of the SFs.

Performance increase as a function of power consumption and number of SVD modes (or channels) is shown in Fig. 6. It can be noticed that SF-SVD coils present a considerable drop in average performance relatively to their generating SO coils: an almost $15 \%$ drop from $\boldsymbol{\Psi}_{\mathrm{DB}}^{1 W}$ to $\boldsymbol{\Psi}_{\mathrm{SVD}}^{1 W, M=1}$, and more than $20 \%$ drop from $\boldsymbol{\Psi}_{\mathrm{DB}}^{100 W}$ to $\boldsymbol{\Psi}_{\mathrm{SVD}}^{100 W, M=1}$. Nevertheless, using a single coil for shimming, average inhomogeneity reduction ranging from $15.8 \%$ to $18.0 \%$ for $\mathcal{T}$ is remarkable. The drop in performance is an expected behavior, as the SF-SVD coils with a small number of modes $M$ can only approximate the actual subject-optimal SFs that originated them.

As more SVD modes are added, further growth in performance is observed. For SF-SVD systems originated from higher target power SO SFs, addition up to the $4^{\text {th }}$ mode shows the most significant increase, which afterwards continues to grow slower but steadily as higher order modes are added. For all systems, the addition of the second mode provides the most significant increase in average performance, 


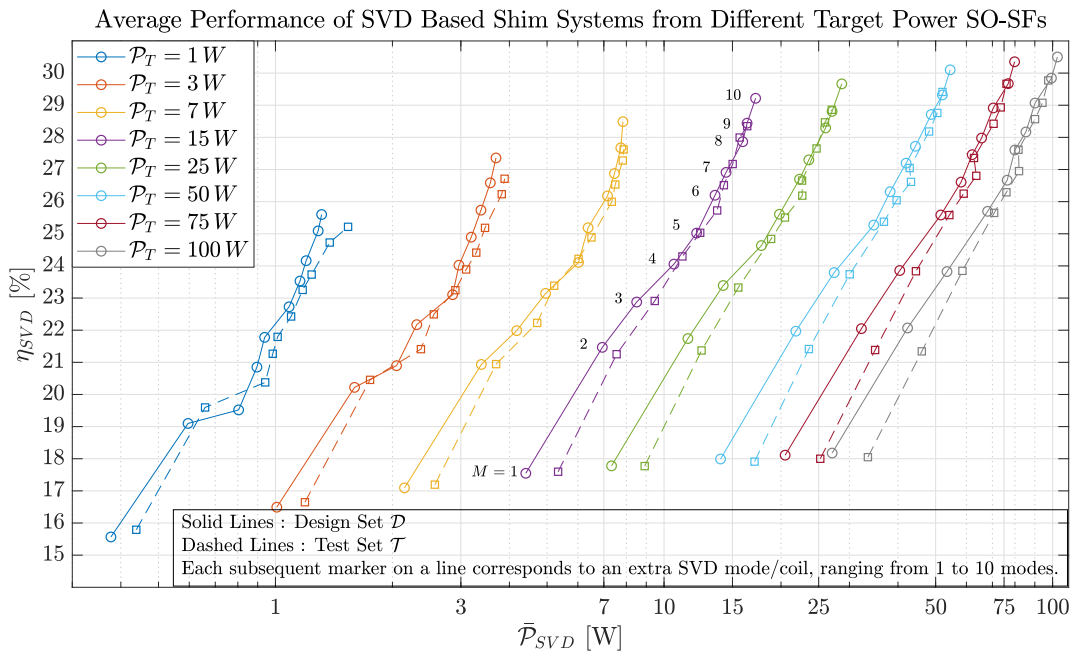

Figure 6: Average performance and power dissipation of different SF-SVD shim systems. Each curve shows the evolution of average performance and average power dissipation of an SVD shim system, generated from SO-SFs targeting a specific power, with each marker (circle or square) representing a different number of SVD modes used to constitute the shim system (as exemplified on the $\mathcal{P}_{T}=15 \mathrm{~W}$ curve). Performances over $\mathcal{D}$ and $\mathcal{T}$ are shown separately on solid and dashed curves, respectively.

between $3.3 \%$ and $4.0 \%$. It can be asserted from the curves, and supported by the mathematical properties of SVD, that the few first modes generate field patterns that are common to most brains, thus having significant effect in reducing the inhomogeneity over the entire database. From the $5^{\text {th }}$ mode onward, the increase in performance as modes are added tends to be lower than $1 \%$, meaning that the coil being added is likely to address very particular field patterns on specific subjects, eventually related to a tilt or rotation of the patient's head, thus correlating much less to the whole database. As more modes are added, the increase in performance over the entire database will tend to be each time smaller, as they are more likely to be addressing particularities of single subjects.

It is observed that, for all designs, performances over $\mathcal{D}$ and $\mathcal{T}$ tend to grow together as more modes are added and present very close values. For the lower power consuming systems, average inhomogeneity reduction on $\mathcal{T}$ is slightly greater than on $\mathcal{D}$, when a low number of modes is used. The difference in performance over unknown maps observed for all systems is sufficiently small to conclude that the SF-SVD method manages to provide coils that adapt to the universe of different subjects. Difference in SF-SVD shimming performance over $\mathcal{D}$ and $\mathcal{T}$ starts to diminish in all cases as the first few modes are added but then rises again, favoring $\mathcal{D}$. This can be explained by the fact that higher order SVD modes tend to address precise characteristics of field patterns of smaller groups of subjects, which are not in $\mathcal{T}$, thus causing greater improvement over $\mathcal{D}$ than over $\mathcal{T}$. In addition, performance on $\mathcal{D}$ at higher target power systems tends to be greater than on $\mathcal{T}$ also for the first few modes, which is caused by the greater field fidelity of the subject-optimal coils at higher power; these are more efficient in attenuating specific details of each subject's fieldmap in $\mathcal{D}$, thus 
introducing stronger bias on the SF-SVD coils.

For all simulated SF-SVD shim systems, slightly greater power dissipation is observed in $\mathcal{T}$. Careful analysis of the power dissipation in $\mathcal{D}$ and test set shows that average power dissipation was biased by an outlier in $\mathcal{T}$, as can be observed in Fig 7 for the $15 \mathrm{~W}$ system. Average power dissipation for $\boldsymbol{\Psi}_{S V D}^{15 W, M=3}$ for instance is 8.5 W(SD:7.6) on $\mathcal{D}$ and $9.5 \mathrm{~W}(\mathrm{SD}: 9.5)$ on $\mathcal{T}$, but an outlier with $57.9 \mathrm{~W}$ is present in $\mathcal{T}$, biasing the average and standard deviation, which, without the outlier subject, are 8.5 W(SD:6.6), evidencing that the SF-SVD system shows equivalent power dissipation behavior whether shimming on $\mathcal{D}$ or $\mathcal{T}$. This outlier was present throughout all eight SF-SVD systems, accounting for the observed deviation in average power dissipation on $\mathcal{T}$.
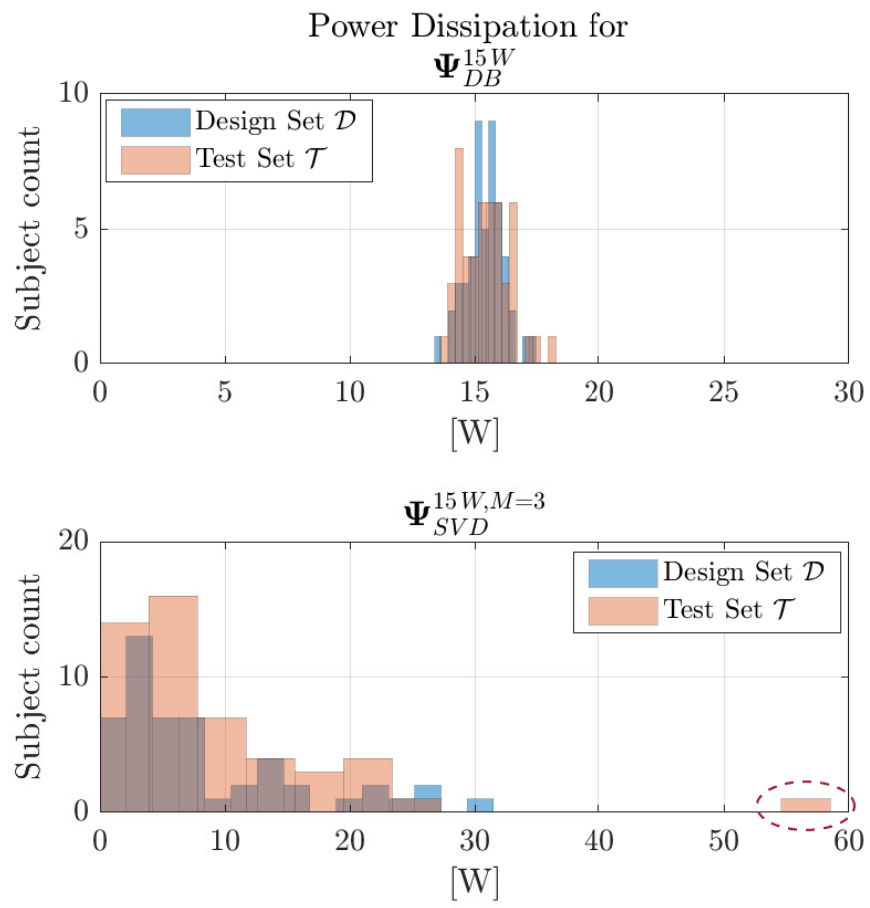

Figure 7: Histogram of power dissipation across subjects for subject-optimal stream functions and SF-SVDs for a $15 \mathrm{~W}$ target system. Outlier subject identified by red ellipse.

It can also be noticed, from Fig. 7, that while the SO coils' power dissipation is very close to the target power set for their design, subsequent SVD application on the SO SF leads to new systems that show a large spread of power dissipation values. Nevertheless, for a fixed number of modes, Fig. 6 shows that the SVD system generated from SO SFs of larger target power will present proportionally larger average power dissipation.

If a low channel count is an important design criterion, SF-SVD systems obtained from SO-SFs of larger target power should be preferred. Although the low power $\boldsymbol{\Psi}_{S V D}^{1 W, M=10}$ shim system would provide $25.2 \%$ average inhomogeneity reduction at a 
low average power dissipation of $1.5 \mathrm{~W}$, the total amount of 10 channels necessary to achieve such performance implies greater hardware complexity and size. On the opposite, the 4-channel $\Psi_{S V D}^{100 W, M=4}$ SF-SVD shim system would provide practically the same level of average inhomogeneity reduction, $25.6 \%$, with less than half the amount of channels. However, power consumption becomes considerably higher, $70.7 \mathrm{~W}$, increasing thermal management complexity of the shim system.

A SF-SVD shim system based on the $\boldsymbol{\Psi}_{S V D}^{15 W}$ SFs seems to provide an appropriate trade-off between performance and power dissipation, since, from this point onwards, SF-SVD systems based on higher power SO SFs will provide marginal performance improvement for a same number of channels.

Cross-validation statistics across the 10 different generations showed 0.97 average performance ratio between $\mathcal{T}$ and $\mathcal{D}, 0.99$ median ratio, 0.09 standard deviation and ranged within 0.80 and 1.09. Results show that independently of the random choice of subjects composing $\mathcal{D}_{j}$, the average inhomogeneity reduction of the SF-SVD shim system over $\mathcal{T}_{j}$ is maintained very close to that over $\mathcal{D}_{j}$, confirming robustness to new subjects.

\subsection{Comparison between SF-SVD and unconstrained SH shimming}

The performance of SF-SVD systems can be compared to what would be achieved when using Spherical Harmonics coils to shim the same database of subjects. For this simulation, the coefficients of each spherical harmonic order (from 0 to the desired SH shim system order) and degree were computed considering ideal coils and no power constraint was imposed on these coils. They were compared to a SF-SVD shim system based on the $\boldsymbol{\Psi}_{S V D}^{15 W}$ SFs, as shown in Fig. 8.

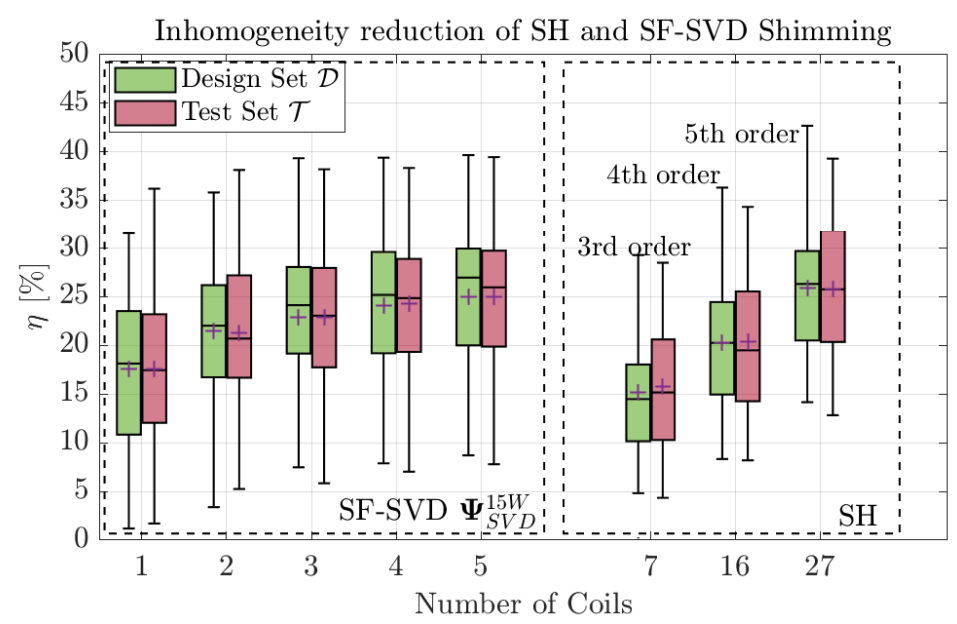

Figure 8: Inhomogeneity reduction comparison between an unlimited power Spherical Harmonics shim system and a 15-W SF-SVD shim system.

Focusing on $\mathcal{T}$, the single channel SF-SVD shimming shows better average performance $(17.6 \%)$ than a 7 -channel full $3^{\text {rd }}$ order Spherical Harmonic based shim system (15.8\%). The 1-channel SF-SVD system's performance between the $25^{\text {th }}$ and $75^{\text {th }}$ percentiles ranges from $12.1 \%$ to $23.2 \%$, while the $3^{\text {rd }}$ order $\mathrm{SH}$ system shows performances from $10.3 \%$ to $20.6 \%$ within the same percentiles. This shows 

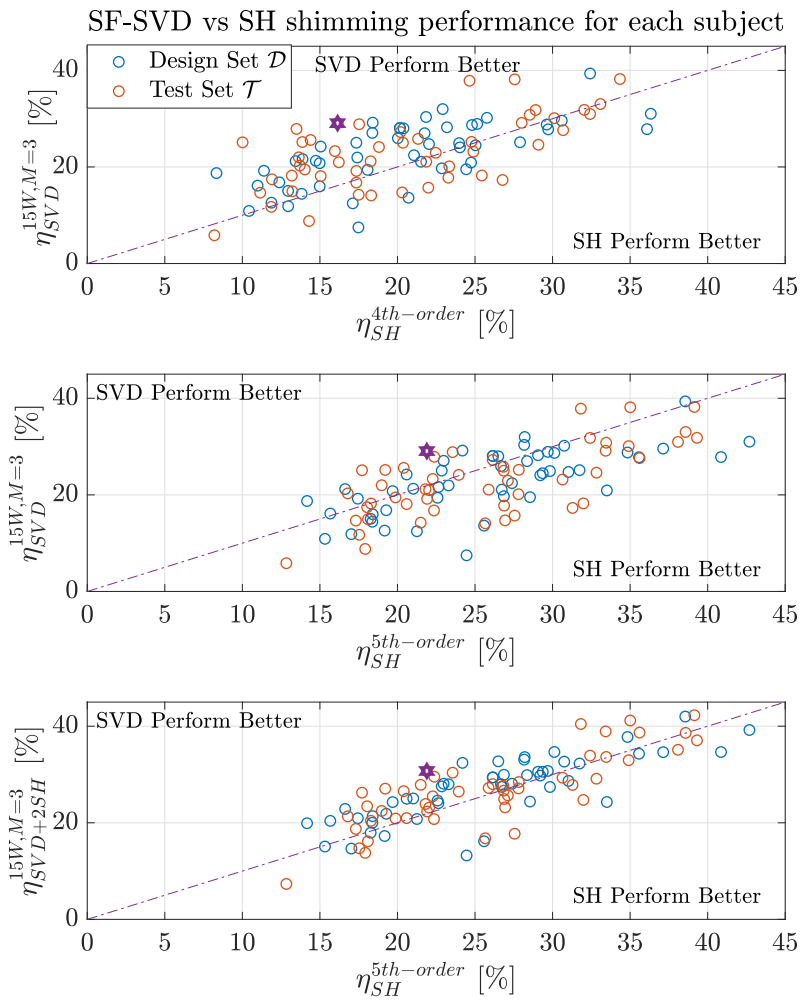

Figure 9: Performance of a 15-W 3-channel SF-SVD shim system vs SH shim systems of different orders for each subject. The SF-SVD system is compared to $4^{\text {th }}$ (top plot) and $5^{\text {th }}$ (middle) order systems. The bottom plot shows its performance against $5^{\text {th }}$ (middle) order when combined with $2^{\text {nd }}$-order refined shimming. The dash-dot line represents equal performance and is present to ease visualization. The purple star indicates the subject shown in the fieldmaps comparison of Fig. 10.

a statistically superior performance for a single SVD channel system against a 7channel SH system. Nevertheless, the 1-channel SF-SVD shim system presents very low performance on some subjects, as shown by the whiskers of its box plots. This situation can be improved by adding extra SVD modes (or channels) to the SFSVD shim system. The 3-channel SF-SVD shim system's average performance of $22.9 \%$ is superior to the $20.4 \%$ performance presented by the 16 -channel full $4^{\text {th }}$ order SH shimming system. In addition, $60 \%$ of subjects in $\mathcal{T}$ present greater inhomogeneity reduction when shimmed by the 3-channel SF-SVD system. This is a remarkable achievement, as a 3-channel system is capable of outperforming a conventional SH system composed by 16-channels, and establishes SF-SVD shim systems as an advantageous alternative to high-order shim inserts. The 27-channel $5^{\text {th }}$ order SH system, however, is harder to outperform, and even the 5-channel SFSVD shim system does not achieve the same average performance. Nevertheless, SF-SVD performance simulations so far considered SF-SVD shimming over the $2^{\text {nd }}$ 


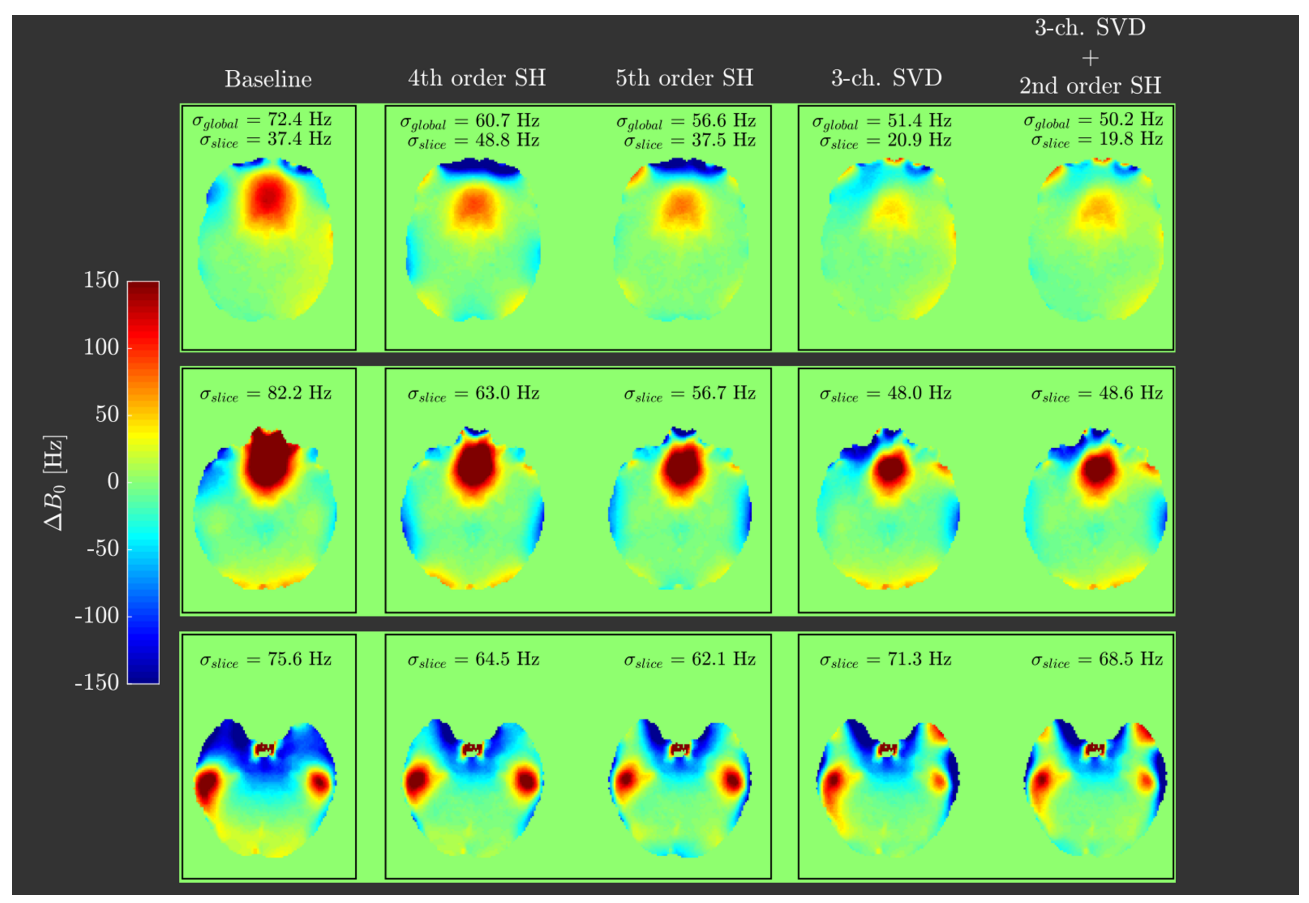

Figure 10: Fieldmap comparison between Baseline, $4^{\text {th }}$-order $\mathrm{SH}, 5^{\text {th }}$-order $\mathrm{SH}, 3$-ch. SF-SVD and mixed 3-ch. SF-SVD $+2^{\text {nd }}$-order SH shims at different axial slices.

order SH shimmed fieldmaps, which implies a shimming pipeline composed by $\mathrm{SH}$ shimming with the scanner's built-in coils and subsequent application of the SF-SVD coil shimming on the resulting map. It would be possible to combine the builtin $2^{\text {nd }}$ order SH system with the 3-channel SF-SVD system in order to compute $\mathrm{SH}$ coefficients and channel currents at the same time, which is shown to improve performances (cf. Fig. 9).

Most subjects in $\mathcal{D}$ and $\mathcal{T}$ present greater inhomogeneity reduction when shimmed by the 3-channel SF-SVD shim system in comparison to the $4^{\text {th }}$ order SH system. As already noted, the $5^{\text {th }}$ order SH shim system outperforms the 3-channel SF-SVD system, with average performances of $25.9 \%$ and $25.8 \%$, for $\mathcal{D}$ and $\mathcal{T}$ respectively. However, by combining the built-in $2^{\text {nd }}$ order shim coils with the 3 -channel SF-SVD system, it can be observed that most subjects present higher inhomogeneity reduction when shimmed by the combined system, with average inhomogeneity reduction of $27.0 \%$ and $26.8 \%$ on $\mathcal{D}$ and $\mathcal{T}$, respectively, with $70 \%$ and $62 \%$ presenting performance when shimmed by the combined SF-SVD+SH system. This mixed system, which would only require a step of characterization of the scanner's shim coils to be implemented, provides better results than a 27-channel high-order shim insert. Improved performances obtained with the combined SF-SVD+SH system come from scanner's shimming software inability to reach optimal shimming of the brain as it takes a larger region (Field of View) into account when computing built-in coil coefficients. Alternatively, a $2^{\text {nd }}$-order re-shim could have been performed on the database before SO-SF computation, but if this improved $2^{\text {nd }}$-order $\mathrm{SH}$ shimming is not implemented by the user, the SF-SVD system obtained from the re-shimmed 

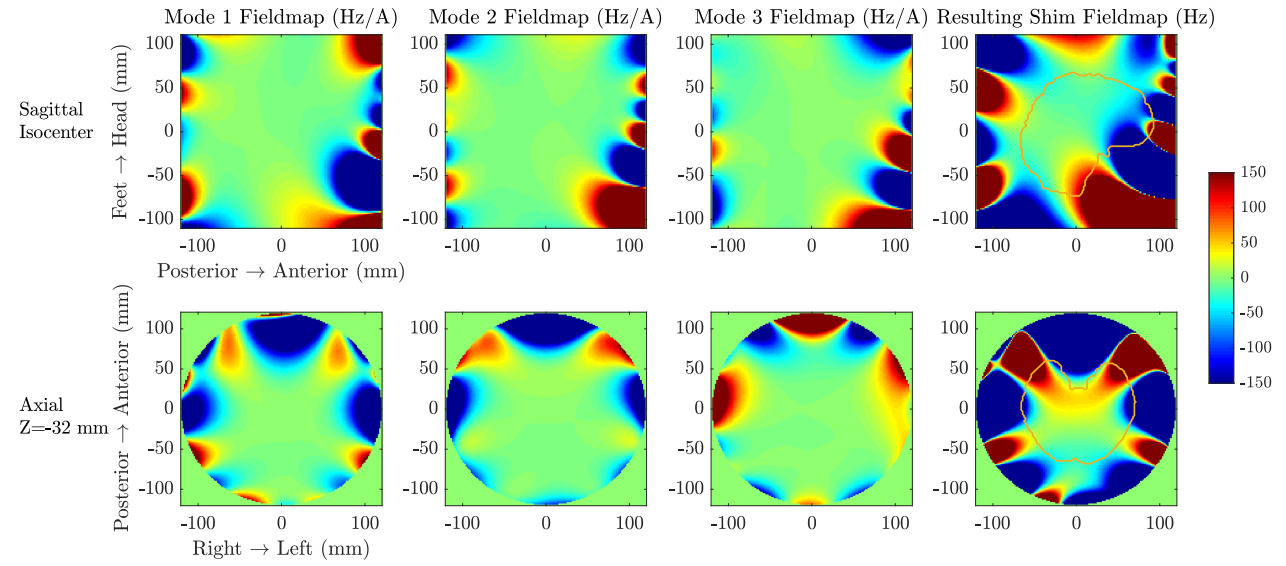

Figure 11: Fieldmap per unit current for SF-SVD modes 1,2 and 3; and resulting magnetic field pattern used for shimming a randomly picked subject using these 3 coils. The brain outline of the particular subject is shown in yellow.

database would under-perform. Thus, by not re-shimming, the designer is free to use the SF-SVD system with its full capacity and eventually improve performance by characterizing the scanner's coils and implementing the combined approach.

A comparison of shimmed fieldmaps is shown in Fig. 10 for a subject in $\mathcal{T}$. The $2^{\text {nd }}$ order shimmed baseline, $4^{\text {th }}$ and $5^{\text {th }}$ order SH shims, 3-ch. SF-SVD and mixed $\mathrm{SF}-\mathrm{SVD}+2^{\text {nd }}$ order $\mathrm{SH}$ shimmed fieldmaps are shown at a few selected slices where intense inhomogeneity is present and the changes on the field patterns can be easily visualized. The areas of stronger inhomogeneity are mainly the frontal and temporal lobes, due to the air cavities located near those regions (sinus, ear canals). The values of global and slice inhomogeneity assert the superior performance of SF-SVD. After either SH or SF-SVD shimming, there are still wide regions containing magnetic field offsets of high intensity (superior to $100 \mathrm{~Hz}$ ), but those regions are reduced in 3-ch. SF-SVD shimming compared to the 16-ch. and 27-ch. SH shimming.

In Fig. 11, the magnetic field distribution generated by each individual SFSVD mode is shown on selected slices. The greater intensity of the magnetic field is observed in the frontal and temporal lobes, as expected. A high degree of symmetry is also remarkable on the 2 first modes, also consistent with the field patterns inside the human brain.

Although MCA shim systems were not simulated in this work for comparison with SF-SVD shim systems, how these systems perform relatively to SH systems can be used as a metric for assessing this feature. As reported in Aghaeifar et al. (2020), non-optimized 48-ch. and 65-ch. regular MCAs show inhomogeneity reduction values gravitating around those obtained by $4^{\text {th }}$ order SH shimming systems. Those are therefore equivalent performances to that of the proposed 3-ch. SF-SVD shim system. Optimized MCAs, a current trend in shim system design, however, can show equivalent performances to $5^{\text {th }}$ order SH systems with 32 channels (Aghaeifar et al. 2020), thus surpassing the system proposed herein. Nonetheless, the very low amount of channels of the SF-SVD system is advantageous for building a compact, easy to control and efficient shim system for the whole human brain at UHF. 


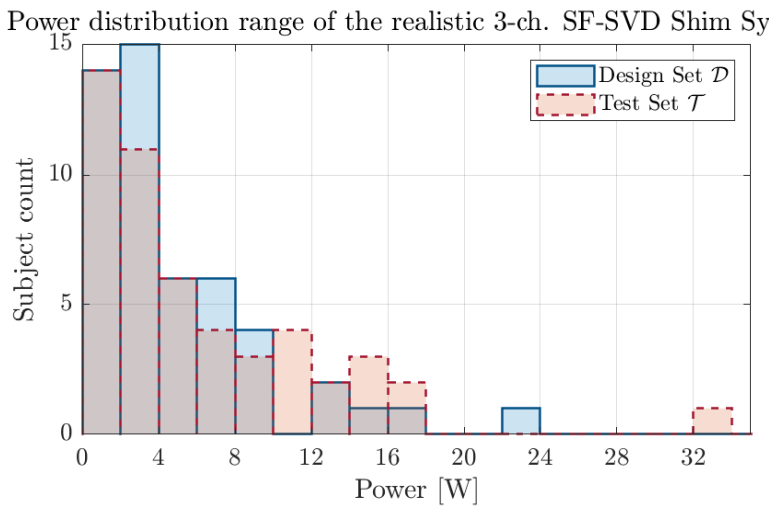

(a) Total power dissipation histogram.

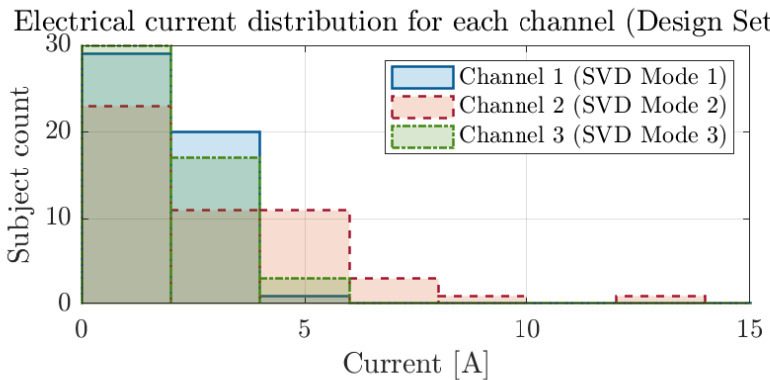

Electrical current distribution for each channel (Test Set $\mathcal{T}$ )

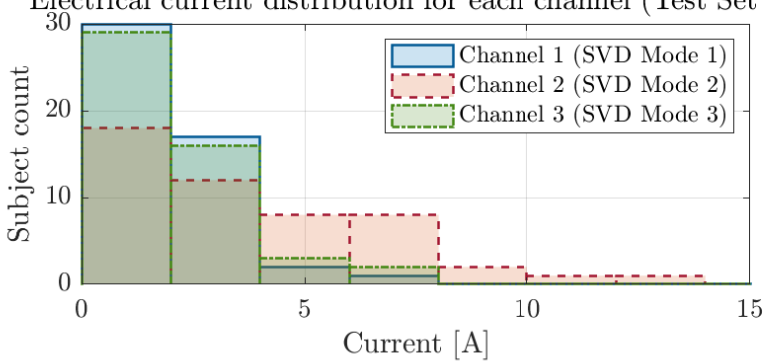

(b) Histogram of current for each of the 3 channels composing the SF-SVD shim system.

Figure 12: Simulated electrical ratings of a realistic 3-ch. SF-SVD shim system applied on the design and test sets.

\subsection{SF-SVD projection onto multiple radii and discretization into windings}

The $\boldsymbol{\Psi}_{\text {SVD }}^{15 W}$ SF-SVD coil projections of second and third layers to higher radii were performed. Projected 3-coil shim system provided practically the same performances as before: $22.8 \%$ vs $22.9 \%$. An increase in power dissipation is nevertheless observed, which is natural as practically the same field intensities are being generated from farther windings, leading to an average power dissipation of $12.3 \mathrm{~W}$ vs $9.5 \mathrm{~W}$ before coil projection to outer radii, with $98 \%$ of subjects in $\mathcal{T}$ under $40 \mathrm{~W}$. At this point we notice that performances of projected SF to outer radii can be maintained the same 
as long as power dissipation is free to increase.

On the other hand, the discretization into realistic windings can be costly in terms of performance. Using $\delta_{w}=2.4 \mathrm{~mm}$, the magnetic fields obtained from the actual windings deviate from SF ideal fields, leading to $16.0 \%$ inhomogeneity reduction for the 3-coil system on both design and test sets, a significant drop compared to the theoretically achievable performances of $22.8 \%$ and $22.9 \%$. This is a drawback of high $\delta_{w}$ and it suggests a need for improving the quality of the discretization into windings. Therefore, the use of smaller wire gauges is required. A lower limit for the wire-gauge would depend on winding technology capabilities and power dissipation rise due to higher resistance of the windings. By decreasing the inter-wire spacing for the first layer to $\delta_{w}^{15 W, M=1}=1.2 \mathrm{~mm}$ (allowing the use of $1 \mathrm{~mm}$ diameter wire), while keeping the $2.4 \mathrm{~mm}$ inter-wire spacing for second and third layers, the gap in performance is reduced, as inhomogeneity reduction for design and test sets are $20.5 \%$ and $20.4 \%$, respectively. This realistic 3-channel SF-SVD design is comparable to the unconstrained 16 -channel $4^{\text {th }}$ order SH system. Simulations showed no improvement when reducing $\delta_{w}$ for the second and third layers, thus the configuration with $1.2 \mathrm{~mm}$ inter-wire spacing for the first layer and $2.4 \mathrm{~mm}$ for both second and third layers should be preferred, as smaller values for $\delta_{w}$ will increase complexity of the windings.

Currents and power dissipation for this realistic SF-SVD insert are shown in Fig. 12. Its average power dissipation is $5.6 \mathrm{~W}$ and maximum power dissipation is $32.5 \mathrm{~W}$, with $98 \%$ of subjects requiring less than $18 \mathrm{~W}$ for shimming. Average currents for each channel are $1.8 \mathrm{~A}, 3.1 \mathrm{~A}$ and $1.9 \mathrm{~A}$ for modes 1,2 and 3 , respectively, on $\mathcal{D}$; and $2.0 \mathrm{~A}, 3.7 \mathrm{~A}$ and $2.0 \mathrm{~A}$ on $\mathcal{T}$. This range of currents could be easily driven by a low-cost, compact, open-source current drive (Arango et al. 2019) (https://www.opensourceimaging.org/project/current-driver-for-local-b0shim-coils/), which can deliver $8 \mathrm{~A}$ per output channel and whose channels could be connected in parallel to eventually supply the max current of $12 \mathrm{~A}$ observed in channel 2 of the SF-SVD shim system.

As a final remark, the $275 \mathrm{~mm}$ outer diameter of the in-house RF coil considered in this study is relatively small compared to most commercial RF coils, which have outer diameters as large as $380 \mathrm{~mm}$. Preliminary simulations of the presented SF-SVD method applied to larger radius coil former $(380 \mathrm{~mm})$ have shown that for keeping performances of a 3-ch. SF-SVD system superior to that of a $4^{\text {th }}$ order SH shim system, average power dissipation grows to $360 \mathrm{~W}$, i.e. 38 times higher than the power dissipation of the small radius system proposed herein. Such high power would require water cooling for heat management, thus increasing complexity of the system despite the low channel count. Alternatively, as shown in Fig. 6, a high performance, low power system could be built if the number of channels is allowed to increase. Indeed, a $20 \mathrm{~W}$ average power SF-SVD shim system with equivalent performances to a $4^{\text {th }}$ order $\mathrm{SH}$ system can be designed if the number of channels is allowed to increase to at least 8 . This highlights the importance of keeping a small radius coil former to achieve high-performance with low power consumption and low channel count.

\section{Conclusion}

From the presented results it can be established that the use of SF Singular Value Decomposition for obtaining a high performance few-channel shim system dedicated to a specific anatomy is promising. Inhomogeneity reduction produced by a 3 -channel SF-SVD-coil insert with $15-20 \mathrm{~W}$ nominal power is equivalent to that achieved by 
a $4^{\text {th }}$-order SH shim insert composed by 16 coils with unlimited power. Moreover, concomitant computation of $2^{\text {nd }}$ SH shim coefficients and SVD currents can improve performance, superior to $5^{\text {th }}$ order $\mathrm{SH}$.

The SF-SVD method applied to 50 brain fieldmaps showed consistence in performance over a test set of subjects of equivalent size, thus confirming that the system can be used for shimming new brain fieldmaps. This was further confirmed by 10-fold cross-validation on the entire 100-brain database.

Provided enough space, the number of coils composing the SF-SVD could be increased, although simulations showed improvements to be small.

To further improve the performance of whole-brain shimming, dedicated shim systems could be designed for populations with specific anatomies, such as large vs small heads, or Asian vs Caucasian head shapes. Alternatively, SF-SVD shim systems could be designed for local shimming in specific areas of the brain, which, for a same power target, might boost performances in the selected region.

However, a drawback is the complexity of the wire patterns for SF-SVD coils, making the fabrication process more laborious, while for $\mathrm{SH}$ coils, several patents depicting relatively simple designs for SH insert fabrication have been proposed, e.g. Punchard (2013). Nevertheless, a 3D model of a possible implementation of a single channel SF-SVD shim system was shown in Fig. 2 for a groove-based design. Alternatively, the system could also be manufactured by cutting the paths through bulk copper.

Although the context of this study is neurological Ultra-High Field MRI, the presented method could easily be applied for anatomies other than the brain.

\section{Acknowledgments}

This work was permitted thanks to a PhD scholarship granted by CEA to Bruno Pinho-Meneses. The authors wish to thank Guy Aubert, Jason Stockmann and Michel Luong for helpful discussions which initiated this work, Michel Bottlaender and Josselin Houenou for providing access to their MRI subjects, Chantal Ginisty, Yann Lecomte, Valerie Berland for MRI acquisitions, and Vincent Gras for the B0map cleanup subroutine.

\section{References}

Adalsteinsson, E., Conolly, S., Xu, H. \& Spielman, D. M. (1999), 'Design of Dedicated Shim Fields', Proc. Int. Soc. Magn. Reson. Med.

Aghaeifar, A., Mirkes, C., Bause, J., Steffen, T., Avdievitch, N., Henning, A. \& Scheffler, K. (2018), 'Dynamic B0 shimming of the human brain at $9.4 \mathrm{~T}$ with a 16 -channel multi-coil shim setup', Magnetic Resonance in Medicine 80(4), 1714-1725.

Aghaeifar, A., Zhou, J., Heule, R., Tabibian, B., Schölkopf, B., Jia, F., Zaitsev, M. \& Scheffler, K. (2020), 'A 32-channel multi-coil setup optimized for human brain shimming at 9.4t', Magnetic Resonance in Medicine 83(2), 749-764.

Arango, N., Stockmann, J. P., Adalsteinsson, E. \& White, J. (2019), 'Ultimate B0 Shim and the Design of Optimal Shim Bases', Proc. Int. Soc. Magn. Reson. Med.

Bringout, G. \& Buzug, T. M. (2015), 'Coil Design for Magnetic Particle Imaging: Application for a Preclinical Scanner', IEEE Transactions on Magnetics 51(2), 1-8.

Can, M. K., Li, P.-Y., Zhou, J., Wu, P.-Y., Li, Y.-T., Ilmoniemi, R. \& Lin, F.-H. (2019), 'Designs of shim coils with distributed currents for 3 t human brain magnetic resonance imaging', Proc. Int. Soc. Magn. Reson. Med.

Chmurny, G. N. \& Hoult, D. I. (1990), 'The Ancient and Honourable Art of Shimming', Concepts in Magnetic Resonance 2(3), 131-149. 
Duyn, J. H. (2012), 'The future of ultra-high field MRI and fMRI for study of the human brain', NeuroImage 62(2), 1241-1248.

Han, H., Song, A. W. \& Truong, T.-K. (2013), 'Integrated parallel reception, excitation, and shimming (iPRES): Integrated Parallel Reception, Excitation, and Shimming', Magnetic Resonance in Medicine 70(1), 241-247.

Jackson, J. (2007), Classical Electrodynamics, 3rd Ed, Wiley India Pvt. Limited.

Jezzard, P. \& Balaban, R. S. (1995), 'Correction for geometric distortion in echo planar images from B0 field variations', Magnetic Resonance in Medicine 34(1), 65-73.

Jia, F., Elshatlawy, H., Aghaeifar, A., Chu, Y., Hsu, Y., Littin, S., Kroboth, S., Yu, H., Amrein, P., Gao, X., Yang, W., LeVan, P., Scheffler, K. \& Zaitsev, M. (2020), 'Design of a shim coil array matched to the human brain anatomy', Magnetic Resonance in Medicine 83(4), 1442-1457.

Juchem, C. \& de Graaf, R. A. (2017), 'B 0 magnetic field homogeneity and shimming for in vivo magnetic resonance spectroscopy', Analytical Biochemistry 529, 17-29.

Juchem, C., Nixon, T. W., McIntyre, S., Boer, V. O., Rothman, D. L. \& de Graaf, R. A. (2011), 'Dynamic multi-coil shimming of the human brain at 7t', Journal of Magnetic Resonance 212(2), 280-288.

Kim, T., Lee, Y., Zhao, T., Hetherington, H. P. \& Pan, J. W. (2017), 'Gradient-echo EPI using a high-degree shim insert coil at 7 T: Implications for BOLD fMRI: GE BOLD fMRI With a Shim Insert Coil at 7 T', Magnetic Resonance in Medicine 78(5), 1734-1745.

Ladd, M. E., Bachert, P., Meyerspeer, M., Moser, E., Nagel, A. M., Norris, D. G., Schmitter, S., Speck, O., Straub, S. \& Zaiss, M. (2018), 'Pros and cons of ultra-high-field MRI/MRS for human application', Progress in Nuclear Magnetic Resonance Spectroscopy 109, 1-50.

Meneses, B. P. \& Amadon, A. (2019a), 'The Dipole Boundary Method : a simple approach to compute stream functions for shim coil design', Proc. Int. Soc. Magn. Reson. Med .

Meneses, B. P. \& Amadon, A. (2019b), 'A novel few-channel coil design for human brain shimming based on stream function Singular', Proc. Int. Soc. Magn. Reson. Med .

Meneses, B. P. \& Amadon, A. (2019c), 'Static-Magnetic-Field Shimming Coil System for Magnetic Resonance Imaging'.

Meneses, B. P., Luong, M. \& Amadon, A. (2019), 'Optimized multi-coil array design for human brain shimming at Ultra-High Field', Proc. Int. Soc. Magn. Reson. Med.

Pan, J. W., Lo, K.-M. \& Hetherington, H. P. (2012), 'Role of very high order and degree B0 shimming for spectroscopic imaging of the human brain at 7 tesla.', Magnetic resonance in medicine 68(4), 1007-1017.

Peeren, G. N. (2003), 'Stream function approach for determining optimal surface currents', Journal of Computational Physics 191(1), 305 - 321.

Pissanetzky, S. (1992), 'Minimum energy MRI gradient coils of general geometry', Measurement Science and Technology 3(7), 667-673.

Poole, M. \& Bowtell, R. (2007), 'Novel gradient coils designed using a boundary element method', Concepts in Magnetic Resonance Part B: Magnetic Resonance Engineering 31B(3), 162-175.

Punchard, W. F. (2013), 'Shim Insert for High-Field MRI Magnets'.

Roméo, F. \& Hoult, D. I. (1984), 'Magnet field profiling: Analysis and correcting coil design', Magnetic Resonance in Medicine 1(1), 44-65.

Salomir, R., de Senneville, B. D. \& Moonen, C. T. (2003), 'A fast calculation method for magnetic field inhomogeneity due to an arbitrary distribution of bulk susceptibility', Concepts in Magnetic Resonance 19B(1), 26-34.

Stockmann, J. P. \& Wald, L. L. (2018), 'In vivo B 0 field shimming methods for MRI at 7 T', NeuroImage 168, 71-87.

Stockmann, J. P., Witzel, T., Keil, B., Polimeni, J. R., Mareyam, A., LaPierre, C., Setsompop, K. \& Wald, L. L. (2016), 'A 32-channel combined RF and B0 shim array for 3t brain imaging', Magnetic resonance in medicine $\mathbf{7 5}(1), 441-451$.

Stockmann, J., Witzel, T., Blau, J., Polimeni, J., Wei, Z., Keil, B. \& Wald, L. (2013), 'Combined shim-rf array for highly efficient shimming of the brain at 7 tesla', Proc. Int. Soc. Magn. Reson. Med 21.

Wald, L. L. (2012), 'The future of acquisition speed, coverage, sensitivity, and resolution', NeuroImage 62(2), 1221-1229. 